\title{
Efecto de la extracción asistida por ultrasonido en la liberación y bioaccesibilidad in vitro de carotenoides, en bebidas elaboradas con mango (Mangifera indica L.) 'Ataulfo' Effect of ultrasound-assisted extraction on the release and in vitro bioaccessibility of carotenoids in mango (Mangifera indica $\mathbf{L}$.) 'Ataulfo'-based beverages
}

G. Mercado Mercado ${ }^{1}$, V. López Teros², E. Montalvo González¹, G. A. González Aguilar ${ }^{3}$ E. Álvarez Parrilla ${ }^{4}$ y S. G. Sáyago Ayerdi ${ }^{1}$

Palabras clave: sub-productos; mango; carotenoides; bioaccesibilidad; bebidas; extracción-asistida por ultrasonido

Keywords: By-products; mango; carotenoids; bioaccessibility; beverage; ultrasound-assisted extraction

Recepción: 04-12-2017 / Aceptación: 20-01-2018

\section{Resumen}

Introducción: Durante el proceso de industrialización del mango se generan cerca del $40 \%$ de subproductos (SP, cáscara y pasta). Estos SP contienen carotenoides que benefician a la salud del consumidor. Por lo tanto, es un tema de interés conocer si los carotenoides pueden ser bioaccesibles. De tal manera, la extracción asistida por ultrasonido (EAU) ha demostrado ser una herramienta útil para aumentar la bioaccesibilidad de diversos compuestos bioactivos. Se propone elaborar bebidas con SP de mango 'Ataulfo' aplicando la EAU y evaluando la bioaccesibilidad de los carotenoides in vitro.

Método: Se elaboraron 4 bebidas: una control $(\mathrm{BC})$ y tres utilizando la EAU (B-EAU1: $\mathrm{X}_{\mathrm{TE}}$ : 30 $\min , \mathrm{X}_{\mathrm{AS}}: 30 \%$, $\mathrm{X}_{\mathrm{C}}: 0.8$; B-EAU2: $\mathrm{X}_{\mathrm{TE}}: 15 \mathrm{~min}, \mathrm{X}_{\mathrm{AS}}: 70 \%$, $\mathrm{X}_{\mathrm{C}}: 0.4$; B-EAU3: $\mathrm{X}_{\mathrm{TE}}: 12$ min, $\mathrm{X}_{\mathrm{AS}}$ : $100 \%$ y $\mathrm{X}_{\mathrm{C}}$ : 1). Se determinó el perfil de los carotenoides por HPLC-DAD y la bioaccesibilidad in vitro de los carotenoides. Se evaluó la liberación de los compuestos en la digestión gástrica (DG) e intestinal (DI); así como la retención de éstos en la fracción indigestible soluble (FIS) e insoluble (FII). De esta manera, se determinó el porcentaje de bioaccesibilidad (\%BA) de los carotenoides. Se determinó la cinética de liberación durante $180 \mathrm{~min}$, estimando los parámetros cinéticos.

Resultados: El contenido de los carotenoides osciló entre 0.01 a $3.29 \mu \mathrm{g} / 100 \mathrm{~mL}$ en las bebidas de mango. Las xantofilas mostraron diferencia significativa en todas las bebidas con EAU en la DI.

\footnotetext{
${ }^{1}$ División de Estudios de Posgrado, Instituto Tecnológico de Tepic, Tepic. E-mail:_sonia.sayago@gmail.com, ssayago@ittepic.edu.mx

${ }^{2}$ Departamento de Ciencias Químico Biológicas, Universidad de Sonora, Hermosillo

${ }^{3}$ Coordinación de Tecnología de Alimentos de Origen Vegetal, Centro de Investigación en Alimentación y Desarrollo A.C., Hermosillo

${ }^{4}$ Departamento de Ciencias Químico Biológicas, Universidad Autónoma de Ciudad Juárez, Ciudad Juárez

(C) Universidad De La Salle Bajío (México)
} 
La EAU tuvo un efecto negativo sobre el contenido de $\beta$-criptoxantina, luteína, y $\beta$-caroteno en BEAU2 y B-EAU3. Por otra parte, el contenido de $\alpha$-caroteno fue similar en todas las bebidas. En la DI, el contenido de $\alpha$-caroteno y $\beta$-caroteno fueron altos en la $\mathrm{BC}$ y los contenidos de luteína y zeaxantina fueron mayores en B-EAU1. El \%BA de luteína, zeaxantina, $\alpha$-caroteno y $\beta$-caroteno en la B-EAU1 fue del 95.63, 56.88, 58.68 y $90.54 \%$, respectivamente. Sin embargo, la $\beta$ criptoxantina y zeaxantina fueron los menos bioaccesibles en BC y B-EAU3 con 96.5 y Zea 92.3 $\%$, respectivamente. La B-EAU1 presentó una mayor velocidad de liberación de luteína, $\beta$ criptoxantina y $\beta$-caroteno.

Discusión o Conclusión: La EAU ocasionó una disminución en el contenido de los carotenoides, debido probablemente a reacciones de isomerización. La liberación de xantófilas y carotenos en las bebidas de mango, pudo deberse a factores asociados a los componentes de la matriz del alimento, interacciones de los carotenoides con otros componentes y el tratamiento al que se sometieron las bebidas. Por otro lado, la EAU favoreció la liberación de estos compuestos. La presencia de carotenoides en la FIS y FII se puede atribuir a la fibra dietética de la cáscara y la goma xantana adicionada. La EAU contribuyó al aumento del \%BA de los carotenos y xantofilas. También, los parámetros de las cinéticas de liberación mostraron que el EAU facilitó el transporte de los carotenoides. Por lo tanto, los parámetros de la cinética de liberación muestran que existen factores que influyen este fenómeno como la acción sinérgica o antagónica entre los carotenoides y otros componentes.

\section{Abstract}

Introduction: During the industrial processing of the mango is wasted close to $40 \%$ of by-products (BP, peel and paste). These BP contain carotenoids that benefit to the consumer's health, such as, the carotenoids. Hence, it is a matter of interest to know if the carotenoids can be bioaccessible. Hence, ultrasound-assisted extraction (UAE) has proven to be a tool to increase their bioaccessibility of diverse bioactive compounds. In this sense, it is proposed to develop beverages where BP were used applying the UAE, evaluating in vitro the bioaccessibility of carotenoids.

Method: Four beverages were developed, a control (CB), and three with UAE (B-UAE1: XET: 30 $\min , \mathrm{X}_{\mathrm{SA}}: 30 \%, \mathrm{X}_{\mathrm{DC}}: 0.8$; B-UAE2: $\mathrm{X}_{\mathrm{ET}}: 15 \mathrm{~min}, \mathrm{X}_{\mathrm{SA}}: 70 \%, \mathrm{X}_{\mathrm{DC}}: 0.4$; B-UAE3: $\mathrm{X}_{\mathrm{ET}}: 12 \mathrm{~min}$, $\mathrm{X}_{\mathrm{SA}}: 100 \%$ and $\left.\mathrm{X}_{\mathrm{DC}}: 1\right)$. The carotenoids profile was analyzed by HPLC-DAD and in the in vitro bioaccessibility. The kinetics of the release of these compounds was evaluated in the gastric 
digestion (GD) and intestinal (ID), as well as, its retention in the soluble indigestible fraction (SIF) and insoluble indigestible fraction (IIF). In this way, it was determined the percentage of bioaccessibility (\%BA) of carotenoids. Finally, it was determined the release kinetics during 180 min, estimating the kinetic parameters (final velocity and constant kinetic).

Results: The carotenoids content were ranged from 0.01 to $3.29 \mu \mathrm{g} / 100 \mathrm{~mL}$ in the mango beverages. The xanthophylls showed significant differences in all beverages with UAE in the ID. The UAE had a negative effect on the $\beta$-cryptoxanthin, lutein, and $\beta$-carotene content in B-UAE2 and B-UAE3. On the other hand, the $\alpha$-carotene content was similar in all beverages. During ID, the $\alpha$-carotene and $\beta$-carotene content were higher in $\mathrm{CB}$ and lutein and zeaxanthin contents were higher in the B-UAE1. The \%BA of lutein, zeaxanthin (Zea), $\alpha$-carotene and $\beta$-carotene in the BUAE1 was 95.63, 56.88, 65.20, and 90.54\%, respectively. Therefore, $\beta \mathrm{Cr}$ and Zea were the most not bioaccessible in $\mathrm{BC}$ and $\mathrm{B}-\mathrm{EAU} 3$, respectively. The B-UAE1 presented a greater rate of release of $\beta$-cryptoxanthin, lutein and $\beta$-carotene.

Discussion or Conclusion: UAE caused a decrease in the carotenoids content due to isomerization reactions. The release of the xanthophylls and carotenes can be due to components of the food matrix, interactions of the carotenoids with other compounds, and the preparation of beverages. However, the UAE favors the release of these compounds. On the other hand, the presence of carotenoids in the SIF and IIF was due to dietary fiber of the peel, and the xanthan gum. In this way, UAE contributes to increase the \%BA of carotenoids. Therefore, the release kinetic parameters show that there are diverse factors that influence this phenomenon such as the synergistic or antagonistic action between the carotenoids and other components.

\section{Introducción}

Las enfermedades crónicas degenerativas son patologías de larga duración, cuyo proceso es generalmente lento; entre las que se incluyen las enfermedades cardiovasculares (ECV), diferentes tipos de cáncer, enfermedades de las vías respiratorias y diabetes mellitus. Todas estas representan un problema que va en un aumento progresivo, debido al envejecimiento de la población y a los estilos de vida actuales que acentúan el sedentarismo y la mala alimentación (Zhang et al., 2015, 1). Actualmente, el $70 \%$ de los mexicanos padecen obesidad, donde cerca de una tercera parte son jóvenes (Colchero et al., 2016, 22). En México, una de las bebidas que contribuye al incremento 
de estos factores de riesgo son las bebidas azucaradas, como los refrescos (Colchero et al., 2016, 33). Por ello, se han implementado alternativas para aumentar el consumo de productos naturales que puedan ayudar a reducir el riesgo de contraer de estas enfermedades. Las frutas y los vegetales son productos naturales que aportan componentes que ejercen beneficios a la salud y se han asociado en la prevención de enfermedades causadas por el estrés oxidativo. Actualmente, existen evidencias científicas que correlacionan un efecto benéfico con el consumo de frutas y vegetales que son ricos en fitoquímicos y la disminución en el desarrollo de las ECV (Zhang et al., 2015, $15)$.

El mango (Mangifera indica L.) pertenece a la familia Anarcadáceas se cultiva en zonas tropicales y sub-tropicales de México, donde crecen diversas variedades de mango, entre ellas la variedad 'Ataulfo'. Durante el proceso de industrialización se utiliza el $60 \%$ del fruto y el $40 \%$ restante son sub-productos (SP), entre los que se encuentran la cáscara (15-20 \%), la pasta (residuo de la obtención del concentrado) y el hueso (60 \%) (Ravani y Joshi, 2013, 690). Estudios previos han demostrado que la cáscara y la pasta son ricos en fitoquímicos, entre ellos fibra dietética, compuestos fenólicos y carotenoides (Blancas-Benítez et al., 2015a, 11; Ajila et al., 2007, 18). Los carotenoides son tetraterpenoides formados por poli-isoprenoides (por ocho unidades) unidas con dobles enlaces conjugados y con ramificaciones de grupos metilo por cada cinco átomos de carbono (Kiokas et al., 2016, 77). Actualmente, se conocen más de 600 carotenoides, que se dividen en carotenos y xantófilas (Kiokas et al., 2016, 47). Los carotenos ( $\alpha$-caroteno y $\beta$-caroteno) y las xantofilas (zeaxantina, $\beta$-criptoxantina, luteína) desempeñan diversas funciones como provitamínicos, antioxidantes, activadores del sistema inmunológico, entre otros (Fiedor y Burda, 2014, 13).

Por otra parte, la extracción asistida por ultrasonido (EAU) ha demostrado ser una tecnología emergente viable para la conservación de alimentos a partir de la inactivación de enzimas, mejorando la calidad sensorial y aumentando la vida de anaquel de diferentes productos (Zou y Jiang, 2016, 24). Sin embargo, existen pocos trabajos que se hayan enfocado sobre el efecto de la EAU en el contenido de carotenoides en los jugos de frutas o subproductos de ellas (Zou y Jiang, 2016, 24; Janiszewska y Sakowski, 2013, 30). En este sentido, aprovechar los SP del mango como ingrediente para la formulación de bebidas podría aumentar el contenido y consumo de carotenoides. Así mismo, la EAU en las bebidas facilitaría la liberación de estos compuestos de los 
Efecto de la extracción asistida por ultrasonido en la liberación y bioaccesibilidad in vitro de carotenoides, en bebidas elaboradas con mango (Mangifera indica L.) 'Ataulfo'

SP y aumentaría su bioaccesibilidad. En este sentido, el presente estudio consiste en evaluar el contenido y bioaccesibilidad de los carotenoides en bebidas a base de SP de mango 'Ataulfo'.

\section{Método}

\section{Obtención de la materia prima}

Los SP (cáscaras y pasta) del mango 'Ataulfo' fueron donados de una empresa procesadora de la ciudad de Tepic, Nayarit, México (Mexifrutas, S.A. de C.V.) y fueron trasladadas inmediatamente al Instituto Tecnológico de Tepic, en donde fueron secados por un proceso convencional a $60{ }^{\circ} \mathrm{C}$ por 15 h y $7 \mathrm{~h}$ para la cáscara y la pasta, respectivamente. Se eligió esta temperatura para conservar los compuestos bioactivos de los SP (Izli et al., 2017, 192). Los SP del mango fueron molidos y almacenados a $-20{ }^{\circ} \mathrm{C}$ hasta su análisis. Los mangos fueron adquiridos en un mercado de la localidad con un estado de madurez de consumo $\left(12.5^{\circ} \mathrm{B}\right)$ y fueron lavados, sanitizados, pelados y se obtuvo la pulpa, que se almacenó a $-20^{\circ} \mathrm{C}$.

\section{Extracción asistida por ultrasonido (EAU) para la preparación de las bebidas de mango}

Todas las bebidas fueron elaboradas con los siguientes ingredientes; agua purificada, cáscara, pasta, pulpa de mango, sacarosa, ácido ascórbico, áloe vera (AgroFresh Food, México) y goma xantana (Drogueria Cosmopolita, México), de acuerdo a lo indicado en la Tabla 1. Los SP del mango se seleccionaron como fuente de fibra dietética. La bebida control (BC) consistió en mezclar los ingredientes mencionados anteriormente en $300 \mathrm{~mL}$ de agua purificada. Posteriormente, se adicionaron $200 \mathrm{~mL}$ más a la mezcla y se agitó hasta quedar totalmente homogenizado en los envases previamente lavados y sanitizados. La preparación de BC se realizó por triplicado, las cuales fueron envueltas con papel aluminio y se almacenaron a temperaturas de refrigeración ( $5 \pm$ $\left.0.1^{\circ} \mathrm{C}\right)$.

En el caso de las bebidas tratadas con la EAU estas fueron preparadas de la misma manera solo que al finalizar la elaboración se empleó un procesador de ultrasonido UP 400S (Hielscher $\mathrm{GmbH}$, Teltow, Germany) con un sonotrodo de 3 a $40 \mathrm{~mm}$ de diámetro y una frecuencia constante de $24 \mathrm{KHz}$. La energía ultrasónica fue controlada por el ajuste de la amplitud de la sonda al sonicator. Posteriormente, el transductor de ultrasonidos (H7 Tip 7, Hielscher, Teltow, Alemania) fue introducido aproximadamente $150 \mathrm{~mm}$ en el interior de cada botella y las bebidas fueron sonicadas en oscuridad a fin de evitar posibles daños inducidos por la luz. El tiempo de extracción 
(TE), la amplitud de sonicación (AS) y el ciclo (C) se utilizaron como variables independientes. Las condiciones óptimas utilizadas en este estudio fueron: el contenido de $\beta$-caroteno (B-EAU1) ( $\mathrm{X}_{\mathrm{TE}}: 30$ min, $\mathrm{X}_{\mathrm{AS}}: 30 \%, \mathrm{X}_{\mathrm{C}}: 0.8$ ); inhibición de los mesófilos en las bebidas de mango (B-EAU2): ( $\left.\mathrm{X}_{\mathrm{TE}}: 15 \mathrm{~min}, \mathrm{X}_{\mathrm{AS}}: 70 \%, \mathrm{X}_{\mathrm{C}}: 0.4\right)$; y la inhibición de las bacterias coliformes (B-EAU3): (X 12 min, $\left.\mathrm{X}_{\mathrm{AS}}: 100 \%, \mathrm{X}_{\mathrm{C}}: 1\right)$. Después de aplicar la EAU, las bebidas se almacenaron a temperaturas de refrigeración $\left(5 \pm 0,1^{\circ} \mathrm{C}\right)$. Las bebidas BC y B- EAUs fueron una suspensión coloidal. Las bebidas (BC y B-EAUs) se envolvieron en papel de aluminio y fueron colocadas sobre hielo para ser transportadas a la Universidad de Sonora en Hermosillo, Sonora e inmediatamente fueron almacenados a $4{ }^{\circ} \mathrm{C}$ hasta su análisis. Las bebidas con la EAU fueron comparados con la BC, que no fue sometida a la EAU (Cuadro 1).

Cuadro 1. Formulación de bebidas preparadas con sub-productos de mango 'Ataulfo'.

\begin{tabular}{lcccc}
\hline & \multicolumn{4}{c}{ Bebidas de mango 'Ataulfo' } \\
\cline { 2 - 5 } & BC' $^{\mathbf{1}}$ & B-EAU1 $^{*}$ & B-EAU2 $^{+}$ & B-EAU3- $^{\circ}$ \\
\hline Cáscara (g) & 1 & 1 & 1 & 1 \\
Pasta (g) & 1 & 1 & 1 & 1 \\
Pulpa (g) & 10 & 10 & 10 & 10 \\
Goma xantana (g) & 0.2 & 0.2 & 0.2 & 0.2 \\
Aloe Vera (g) & 0.4 & 0.4 & 0.4 & 0.4 \\
Azúcar comercial (g) & 12 & 12 & 12 & 12 \\
Ácido ascórbico (g) & 0.4 & 0.4 & 0.4 & 0.4 \\
Agua (mL) & 500 & 500 & 500 & 500 \\
\hline
\end{tabular}

1: Bebida de mango control. B- EAU: bebidas de mango tratadas con extracción asistida por ultrasonido (EAU): *Condición óptima de extracción para $\beta$-caroteno en cáscara y pasta $\left(\mathrm{X}_{\mathrm{TE}}\right.$ : 30 min, $\left.\mathrm{X}_{\mathrm{A}}: 30 \%, \mathrm{X}_{\mathrm{C}}: 0.8\right) .{ }^{+}$Condición óptima para la inhibición de mesófilos en bebidas de mango $\left(\mathrm{X}_{\mathrm{TE}}: 15 \mathrm{~min}, \mathrm{X}_{\mathrm{A}}: 70 \%, \mathrm{X}_{\mathrm{C}}\right.$ : 0.4). Condición óptima para la inhibición de coliformes totales $\left(\mathrm{X}_{\mathrm{TE}}: 12 \mathrm{~min}, \mathrm{X}_{\mathrm{A}}: 100 \%, \mathrm{X}_{\mathrm{C}}: 1\right)$.

\section{Extracción de carotenoides en las bebidas de mango}

La extracción de los carotenoides de las bebidas del mango se realizó siguiendo el método descrito por Qin $(2008$, 78). Brevemente, $200 \mu \mathrm{L}$ de cada bebida se homogenizó con una disolución salina $(500 \mu \mathrm{L}, 0,85 \% \mathrm{w} / \mathrm{v})$, y cloroformo-metanol $(3000 \mu \mathrm{L}, 2: 1 \mathrm{v} / \mathrm{v})$ y se utilizó equinenona como estándar interno $(100 \mu \mathrm{L})$, se agitaron por $30 \mathrm{~s}$ a temperatura ambiente $\left(25^{\circ} \mathrm{C}\right)$. Después, se centrifugaron $\left(3500 \mathrm{rpm} \mathrm{a} 4{ }^{\circ} \mathrm{C}\right.$ por $10 \mathrm{~min}$ ) y el extracto orgánico fue recuperado. Posteriormente, 
el residuo se lavó con $3000 \mu \mathrm{L}$ de hexano (BHT $0.25 \%$ w/v) y se repitió el procedimiento anterior. Cada uno de los extractos se llevaron a sequedad por arrastre de nitrógeno gaseoso a $40{ }^{\circ} \mathrm{C}$ (Organomation, N-Evap 111, USA) para ser re-suspendidos en $200 \mu \mathrm{L}$ de etanol (BHT $0.25 \%$

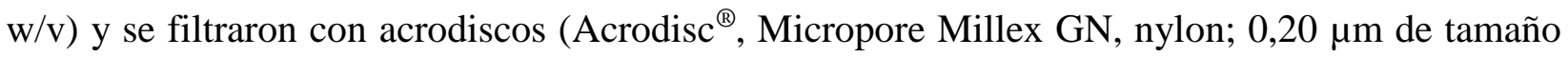
de poro de $13 \mathrm{~mm}$ de diámetro), se colocaron en frascos viales ámbar para cromatografía de 1.5 $\mathrm{mL}$ (Thermo Scientific Fsher) e inmediatamente fueron analizados por HPLC-DAD.

\section{Análisis de carotenoides por HPLC-DAD}

El análisis de los carotenoides por HPLC-DAD se realizó siguiendo el método descrito por Qin $(2008$, 78). Los extractos de los carotenoides fueron analizados en un cromatógrafo de líquidos de alta presión Agilent 1200 (Agilent 1200 Series $^{\circledR}$, USA), equipado con una bomba cuaternaria, un inyector de muestra automática, un detector de arreglo de diodos (DAD) y equipado con una columna $\mathrm{C}_{18}$ Alltima $\left(3,0 \times 150 \mathrm{~mm}, 5 \mu \mathrm{m}\right.$ de partículas, Thermo Scientific ${ }^{\circledR}$, Wilmington, USA) por un sistema binario de dos solventes en diferentes proporciones. Se utilizaron dos eluentes para el análisis, el eluente A fue metanol/metil ter-butil éter/ acetato de amonio 0.03M en agua (85:12:3 $\mathrm{v} / \mathrm{v} / \mathrm{v})$ y el eluente B fue metanol/metanol ter-butil éter/acetato de amonio 0.03M (8:90:2 v/v/v). El gradiente utilizado fue $0 \mathrm{~min}, 0 \% \mathrm{~B} ; 21 \mathrm{~min}, 45 \% \mathrm{~B} ; 22 \mathrm{~min}, 55 \% \mathrm{~B} ; 33 \mathrm{~min}, 95 \% \mathrm{~B}$; después se mantuvo a $95 \%$ B, hasta los 39 min; y finalmente, un ciclo de acondicionamiento de 20 min con las condiciones iniciales para el siguiente análisis. El flujo de elución fue de $0.4 \mathrm{~mL} \mathrm{~min}^{-1}$ y el tiempo total de la corrida fue $60 \mathrm{~min}$. El volumen de inyección de las muestras fue de $10 \mu \mathrm{L}$. La cuantificación individual de los compuestos se realizó con los métodos de calibración utilizando el estándar externo (equinenona) y el estándar añadido a las muestras para obtener el porcentaje de recuperación de equinenona. Para el cálculo de los porcentajes de recuperación (\%R) se procedió en inyectar en el HPLC-DAD cada extracto a una concentración conocida de equinenona y se obtuvo un área para cada carotenoide a medir. Luego, las áreas de los compuestos fueron restadas con el área de equinenona externo y con este resultado se calculó la concentración obtenida de cada carotenoide. El porcentaje de recuperación se calculó de acuerdo con la Ec. 1.

$$
\begin{array}{ll}
\% R=\frac{C_{E o}}{C_{E a}} & \text { Ec. } 1
\end{array}
$$


Donde el \% R es el porcentaje de recuperación. $\mathrm{C}_{\mathrm{Eo}}$ es la concentración de equinenona obtenido. $\mathrm{C}_{\mathrm{Ea}}$ es la concentración de equinenona agregada.

Los carotenoides analizados en las bebidas de mango fueron: $\alpha$-caroteno $(\alpha \mathrm{C})$ y $\beta$-caroteno $(\beta C)$; y las xantofilas: $(\beta$-criptoxantina $(\beta \mathrm{Cr})$, Luteina (Lut) y zeaxantina (Zea). Los picos se compararon con los tiempos de retención $\left(t_{R}\right)$ y los espectros de absorción de UV/Vis de los estándares. Los carotenoides fueron detectados a $450 \mathrm{~nm}$ y los resultados fueron expresados como $\mu \mathrm{g}$ de carotenoide/100 $\mathrm{mL}$ de la bebida.

\section{Bioaccesibilidad in vitro de carotenoides}

La digestión gastrointestinal in vitro se realizó siguiendo el método descrito por Saura-Calixto et al. (2000) con algunas modificaciones. Las bebidas fueron digeridas en dos fases secuenciales: digestión gástrica (DG) e intestinal (DI). Para este análisis se colocaron 6 muestras de cada bebida, los cuales se tomaron duplicados en cada etapa de la digestión para poder ser analizadas, tal como se describe a continuación. Se midieron $100 \mathrm{~mL}$ de cada bebida y se les añadió $10 \mathrm{~mL}$ de la solución $\mathrm{HCl}-\mathrm{KCl}(0.2 \mathrm{M}, \mathrm{pH}$ 1.5). Posteriormente, se agregó $200 \mu \mathrm{L}$ de una disolución de pepsina (P-7000, $\geq 250$ unidades/mg Sigma-Aldrich Chemical Co., St Louis MO, USA; $300 \mathrm{mg} / \mathrm{mL}$ ) y se incubaron a $40^{\circ} \mathrm{C}$ por $1 \mathrm{~h}$ con agitación constante. Pasado el tiempo, los $100 \mathrm{~mL}$ de cada bebida por duplicado de cada tratamiento fueron centrifugados a $3000 \mathrm{rpm}$ por 15 min a $5{ }^{\circ} \mathrm{C}$ y el sobrenadante se recuperó; se añadió nitrógeno líquido por $5 \mathrm{~s}$ y se almacenaron a $-45^{\circ} \mathrm{C}$, con la finalidad de evitar la oxidación de los carotenoides. Este paso se llamó digestión gástrica (DG). Por otro lado, otro lote de muestra continuó el proceso de digestión, a los tubos se les agregó $5 \mathrm{~mL}$ de amortiguador de fosfato $(0.1 \mathrm{M}, \mathrm{pH} 7.5)$ y $1000 \mu \mathrm{L}$ de pancreatina porcina $(5 \mathrm{mg} / \mathrm{mL}$ de amortiguador de fosfato 0.1 M, pH 7.5, P-1750 Sigma Aldrich,) y se incubaron por $6 \mathrm{~h}$ a $37^{\circ} \mathrm{C}$ con agitación constante. Posteriormente, se agregaron $9 \mathrm{~mL}$ del tampón Tris-maleato (0.1 M, pH 6.9) y se mezclaron con la disolución de $\alpha$-amilasa (A-3176 Type VI-B, Sigma Aldrich). La solución se colocó en un baño a $37^{\circ} \mathrm{C}$ por $16 \mathrm{~h}$ en agitación constante. Se tomó una muestra considerada como digestión intestinal (DI) y se almacenaron en las mismas condiciones mencionadas anteriormente.

Por otro lado, otro lote de muestras procedente de la digestión intestinal se centrifugó y los sobrenadantes de los duplicados restantes se mezclaron con dos lavados con $5 \mathrm{~mL}$ de agua destilada

y fueron transferidos a las bolsas de diálisis (12,000-14,000 de corte de peso molecular; tubo de diálisis Visking, Sigma Aldrich). Las muestras se dializaron a $25^{\circ} \mathrm{C}$ por $48 \mathrm{~h}$. Finalizado el proceso, 
el contenido retenido de la bolsa de diálisis se almacenó en las mismas condiciones, esta fracción se le llamó fracción indigestible soluble (FIS). Por otro lado, el residuo se dejó secar durante 24 h y se almacenó $45^{\circ} \mathrm{C}$, nombrando a esta fracción como fracción indigestible insoluble (FII). A cada una de las fracciones obtenidas se les extrajeron los carotenoides y a partir de los resultados obtenidos se determinó el porcentaje de la bioaccesibilidad (\%BA) de cada carotenoide utilizando la Ec. 2.

$$
\% B A=\frac{(D I-F I S)}{(F I S+F I I)} \quad \text { Ec. } 2
$$

Donde el \%BA es el porcentaje de bioaccesibilidad ( $\alpha \mathrm{C}, \beta \mathrm{C}, \beta \mathrm{Cr}$, Lut y Zea), DI son los caroteoides liberados en la digestión intestinal, FIS son los carotenoides determinados en la fracción indigestible soluble, FII son los carotenoides determinados en la fracción indigestible insoluble.

El porcentaje carotenoides no bioaccesibles (\%NBA) fue calculado a partir de la Ec. 3:

$$
\% N B A=100-\% B A \quad \text { Ec. } 3
$$

\section{Cinética de liberación de los carotenoides}

La cinética de liberación de los carotenoides se determinó siguiendo el método reportado por Blancas-Benítez et al. (2015b, 57) con algunas modificaciones. Se tomaron $100 \mathrm{~mL}$ de cada bebida y se mezclaron con $10 \mathrm{~mL}$ de amortiguador de fosfatos $(0.05 \mathrm{M}, \mathrm{pH} 1.5)$; el pH se ajustó a 1.5. A continuación se añadió $200 \mu \mathrm{L}$ de la disolución de pepsina (P-7000, polvo $\geq 250$ unidades/mg de Sigma-Aldrich; $300 \mathrm{mg} / \mathrm{mL}$ ) y se incubó a $37{ }^{\circ} \mathrm{C}$ durante $1 \mathrm{~h}$. Posteriormente, se ajustó el pH de las bebidas a 6.9 con $20 \mathrm{~mL}$ de amortiguador de fosfatos $0.05 \mathrm{M}(\mathrm{pH}$ 6.9) y se pusieron en bolsas de diálisis de celulosa (12,000-14,000 de corte de peso molecular; tubo de diálisis Visking, Sigma Aldrich), previamente hidratadas en $100 \mathrm{~mL}$ buffer fosfato $0,05 \mathrm{M}$ (pH 6.9) durante $10 \mathrm{~min}$. Se añadió $1000 \mu \mathrm{L}$ de la disolución de la $\alpha$-amilasa pancreática (A-6255, Sigma-Aldrich; Sigma 110 $\mathrm{U} / \mathrm{mL}, 40 \mu 1 / 7 \mathrm{~mL}$ ), ajustando el volumen a $30 \mathrm{~mL}$. Las bolsas de diálisis se colocaron en recipientes con $200 \mathrm{~mL}$ de tampón de fosfatos $(0.05 \mathrm{M}, \mathrm{pH} 6.9)$, previamente estabilizados a $37^{\circ} \mathrm{C}$ y en contante agitación. Se tomaron alícuotas de $1000 \mu \mathrm{L}$ del medio externo (tampón de fosfato $0.05 \mathrm{M}, \mathrm{pH} 6.9,37^{\circ} \mathrm{C}$ ) en intervalos de $30 \mathrm{~min}$ por $3 \mathrm{~h}$ las cuales fueron congeladas en nitrógeno líquido y almacenados a $-45^{\circ} \mathrm{C}$ hasta su análisis. Los carotenoides se extrajeron de cada alícuota 
para ser cuantificados y a partir de estos resultados se determinó la velocidad inicial de liberación con la Ec. 4:

$$
v_{o}=\frac{\left(C_{1}-C_{0}\right)}{\left(t_{1}-t_{0}\right)} \quad \text { Ec. } 4
$$

Donde: $v_{o}$ es la velocidad inicial de liberación de los carotenoides en tiempo específico durante la digestión in vitro $(\mathrm{mg} / \mathrm{min}), C_{1}-C_{0}$ es la diferencia de concentración entre la concentración específica con la concentración inicial $(\mathrm{mg} / \mathrm{g} \mathrm{db}) \mathrm{y}_{1}-\mathrm{t}_{0}$ es la diferencia de tiempo entre el tiempo específico y el tiempo inicial (min).

La velocidad final $(V f)$ y la constante de cinética $(k)$ de $\alpha \mathrm{C}, \beta \mathrm{C}, \beta \mathrm{Cr}$, Lut y Zea fueron determinadas a partir de las Ec. 5 y 6 :

$$
\begin{array}{rr}
V f=\sum\left(\frac{\Delta C}{\Delta t}\right) & \text { Ec. } 5 \\
k=\frac{V f}{[C]} & \text { Ec. } 6
\end{array}
$$

Donde, $\Delta C$ es la diferencia de concentración, $\Delta t$ es la diferencia del tiempo entre el tiempo específico y el tiempo inicial, $V f$ es la velocidad final de los carotenoides liberados durante la cinética de liberación in vitro, $t$ es tiempo.

Una vez determinada la velocidad de liberación de los carotenoides se ajustaron a modelos de orden cero (Ec. 7) o de primer orden (Ec. 8):

$$
\begin{array}{lc}
A=A_{0}-k t & \text { Ec. } 7 \\
\frac{A}{A_{0}}=e^{(-k t)} & \text { Ec. } 8
\end{array}
$$

Donde: $A_{0}$ es el parámetro estimado, el sub-índice ${ }_{0}$ indica el valor inicial, $t$ es el tiempo (min) y $k$ es la constante cinética en función del tiempo.

\section{Análisis estadístico}

Se realizó un análisis de varianza (ANOVA) de una sola vía, con el fin de determinar el efecto de la EAU sobre los carotenoides $(\alpha=0.05)$. La prueba posterior utilizada fue una comparación 
múltiple de las medias aplicando la prueba de Tukey. También, se realizó una prueba de t-Student para poder comparar entre la DG y DI, así como FIS y FII. Los datos fueron procesados por el programa estadístico Statistica 10 (Stat Soft. Inc., Tulsa, OK, USA).

\section{Resultados y Discusión}

\section{Elaboración de las bebidas de mango}

Las bebidas fueron elaboradas con pulpa, cáscara y pasta de mango 'Ataulfo', los cuales son ricos en carotenoides. En estudios previos de nuestro grupo se cuantificó el contenido de $\beta \mathrm{C}$ en la cáscara y la pasta de esta variedad, dando resultados de $1.29 \pm 0.12 \mathrm{mg} / \mathrm{g}$ p.s y $0.56 \pm 0.007 \mathrm{mg} / \mathrm{g}$ p.s., respectivamente (Datos no publicados). Así mismo, se determinó la condición óptima de la EAU, la cual fue $\mathrm{X}_{\mathrm{TE}}: 30$ min, $\mathrm{X}_{\mathrm{AS}}: 30 \%$, $\mathrm{X}_{\mathrm{C}}: 0.8$ para ambos $\mathrm{SP}$. Bajo estas condiciones el contenido de carotenoides se incrementó a $19.13 \mathrm{mg} / \mathrm{g}$ p.s. para la cáscara y $6.60 \mathrm{mg} / \mathrm{g}$ p.s para la pasta. Además, estos SP son ricos en fibra dietética (14.97\% en pasta y $41.34 \%$ en cáscara) (Blancas-Benítez et al. (2015b, 57). Por tal motivo, elaborar una bebida a base de estos SP de mango 'Ataulfo' pueden aportar ciertos beneficios a la salud debido a los compuestos bioactivos que presentan y que pueden tener actividad anti-proliferativa, anti-inflamatoria, inmunoreguladora, así como inhibir la peroxidación lipídica y aumentar la citotoxicidad de los linfocitos T y B en la sangre (Lauricella et al., 2017, 100; Lin et al. 2016, 22). También, se decidió adicionar aloe vera a las bebidas, ya que puede contribuir en diversas propiedades como la actividad del sistema nervioso central, actividad angiogénica, inmunoreguladora, antimicrobiana y gastroprotectora por su alto contenido de micronutrientes esenciales ( $\mathrm{Na}, \mathrm{Ca}, \mathrm{Mg}$ y $\mathrm{K}$ ), así como vitaminas, compuestos fenólicos, antraquinonas, mono y polisacáridos (pectina, hemicelulosa), esteroles, saponinas, aloína, aloe emodina, entre otros (Nandal y Bhardwaj, 2012, 84). Además, el aloe vera ayuda a conservar ciertos alimentos, esto debido a su ordenamiento molecular e isodiamétrica manteniendo sus propiedades sensoriales (Radi et al., 2017, 38). Sin embargo, su alto contenido de fibra dietética puede reducir la absorción de los carotenoides. Por tal motivo, se empleó la EAU debido a que esta tecnología puede romper las interacciones de la fibra dietética dejando más bioaccesibles a los compuestos que se encuentran dentro de la matriz de la fibra dietética (Carail et al., 2015, 24). 


\section{Efecto de la EAU sobre el contenido de carotenoides en las bebidas de mango 'Ataulfo'}

Al realizar los análisis cromatográficos de las bebidas de mango (BC y B-EAU), se identificaron y cuantificaron tres xantófilas $(\beta \mathrm{Cr}$, Lut y Zea) y dos carotenos $(\alpha \mathrm{C}$ y $\beta \mathrm{C})$, tal como se reporta en el Cuadro 2, donde se observa que en la BC, la Lut fue la que se encontró en mayor cantidad (3.29 $\mu \mathrm{g} / 100 \mathrm{~mL})$, seguido de $\alpha \mathrm{C}(1.11 \mu \mathrm{g} / 100 \mathrm{~mL}), \beta \mathrm{Cr}(0.21 \mu \mathrm{g} / 100 \mathrm{~mL})$ y Zea $(0.01 \mu \mathrm{g} / 100 \mathrm{~mL})$. Destaca el resultado de $\alpha \mathrm{C}$, que solamente había sido identificado en mango en las variedades 'Gedong', 'Manalagi', 'Golek', 'Harum manis', 'Black-gold' e 'Indramayn' (Khoo et al., 2011, 200). Nuestros resultados concuerdan con estudios previos, donde se ha reportado que la Lut es la xantófila que se encuentra en mayor concentración en la pulpa de mango (Chen et al., 2004, 16).

Cuadro 2. Contenido de carotenos y xantofilas en bebidas de mango 'Ataulfo'.

\begin{tabular}{|c|c|c|c|c|}
\hline & \multicolumn{4}{|c|}{ Bebidas de mango (Mangifera indica L.) 'Ataulfo' $(\mu \mathrm{g} / 100 \mathrm{~mL})$} \\
\hline & $\mathbf{B C}^{\mathbf{1}}$ & B-EAU1 & B-EAU2 & B-EAU3 \\
\hline \multicolumn{5}{|c|}{ Caroteno } \\
\hline$\alpha \mathrm{C}$ & $1.11 \pm 0.03^{\mathrm{a}}$ & $1.09 \pm 0.03^{\mathrm{a}}$ & $1.13 \pm 0.03^{\mathrm{a}}$ & $1.16 \pm 0.03^{\mathrm{a}}$ \\
\hline$\beta C$ & $0.16 \pm 0.003^{\mathrm{a}}$ & $0.14 \pm 0.002^{\mathrm{b}}$ & $0.07 \pm 0.001^{\mathrm{c}}$ & $0.07 \pm 0.001^{\mathrm{c}}$ \\
\hline \multicolumn{5}{|c|}{ Xantofilas } \\
\hline$\beta \mathrm{Cr}$ & $0.21 \pm 0.004^{\mathrm{b}}$ & $0.37 \pm 0.007^{\mathrm{a}}$ & $0.16 \pm 0.003^{\mathrm{d}}$ & $0.18 \pm 0.003^{\mathrm{c}}$ \\
\hline Lut & $3.29 \pm 0.16^{\mathrm{a}}$ & $2.50 \pm 0.12^{\mathrm{b}}$ & $1.44 \pm 0.07^{\mathrm{d}}$ & $1.50 \pm 0.07^{\mathrm{c}}$ \\
\hline Zea & $0.01 \pm 0.003^{\mathrm{b}}$ & $0.01 \pm 0.0003^{\mathrm{b}}$ & $0.01 \pm 0.0003^{b}$ & $0.02 \pm 0.0003^{\mathrm{a}}$ \\
\hline
\end{tabular}

:BC: bebida sin tratamiento. B- EAU: bebida con tratamiento de ultrasonido-asistido: ${ }^{1} \mathrm{X}_{\mathrm{TE}}$ : $30 \min , \mathrm{X}_{\mathrm{A}}: 30 \%, \mathrm{X}_{\mathrm{C}}: 0.8 .{ }^{2} \mathrm{X}_{\mathrm{TE}}: 15 \min , \mathrm{X}_{\mathrm{A}}: 70 \%, \mathrm{X}_{\mathrm{C}}: 0.4 .{ }^{3} \mathrm{X}_{\mathrm{TE}}: 12 \min , \mathrm{X}_{\mathrm{A}}: 100 \%, \mathrm{X}_{\mathrm{C}}: 1$. $\alpha$-caroteno $(\alpha \mathrm{C}), \beta$-caroteno ( $\beta \mathrm{C}), \beta$-criptoxantina $(\beta \mathrm{Cr}$ ), luteina (Lut) y zeaxantina (Zea). Los valores representan media \pm desviación estándar de tres repeticiones. Diferentes letras representan diferencia significativa $(\alpha=0.05)$ entre los diferentes tratamientos y el control.

El contenido de carotenoides en diferentes frutas oscila entre $0.8 \mu \mathrm{g} / 100 \mathrm{~mL}$ a $151.4 \mu \mathrm{g} / 100 \mathrm{~mL}$ (Aschoff et al., 2014, 213). Nuestros resultados fueron similares a los reportados en jugos con leche de soya tratados con altas presiones hidrostáticas (Cilla et al., 2012, 324). Sin embargo, los resultados obtenidos en esta investigación fueron menores a los reportados en la pulpa liofilizada de mango 'Tommy Atkins' (Petry y Mercadante, 2018, 335).

$\mathrm{Al}$ aplicar la EAU el contenido de los carotenos y las xantofilas cambiaron con respecto al $\mathrm{BC}$ (Cuadro 2), en donde se apreció un efecto negativo de la EAU sobre el contenido de Lut y $\beta \mathrm{C}$ y en menor medida $\beta \mathrm{Cr}$ en las B-EAU2 y B-EAU3. Esto es debido al efecto de la cavitación sobre 
la estructura de estos compuestos (Song et al., 2015, 130). Song et al. (2015) observaron que los carotenoides sufren cambios estructurales cuando se encuentran en soluciones acuosas. Carail et al. (2015) reportaron que los carotenoides son inestables a altas amplitudes de sonicación (Carail et al., 2015, 274). Otros autores han reportado que los carotenoides se isomerizan durante el proceso de cavitación (Song et al., 2015, 134). Este cambio estructural (trans a cis) puede facilitar a que las xantofilas sean solubilizadas y ser absorbidas con mayor facilidad (Periago et al., 2001, 462). Por otro lado, el contenido de $\alpha \mathrm{C}$ y Zea fue similar en todas las bebidas. El contenido de $\alpha \mathrm{C}$ no tuvo diferencia significativa ( $\mathrm{p}>0.05$ ) entre las bebidas. Esto se debe que el $\alpha \mathrm{C}$ es un isómero que se forma a partir del 15-cis- $\beta$-caroteno durante el almacenamiento (Ornelas-Paz et al., 2008, 30; Marx et al., 2003, 45). El contenido de $\beta C$ presentó diferencia significativa $(\mathrm{p}<0.05)$ en la BEAU1, respecto a la BC (Cuadro 2). Se observó una disminución drástica en el contenido de $\beta C$ en las muestras B-EAU2 y B- EAU3, lo cual parece indicar que la amplitud de extracción tiene un efecto importante en la estabilidad del $\beta C$. También, la $\beta C r$ tuvo la misma tendencia por lo que las condiciones del EAU influyeron en la liberación de estos compuestos (Goula et al., 2017, 254).

\section{Efecto de la EAU sobre la bioaceesibilidad in vitro de los carotenoides}

La liberación de los carotenos $(\alpha \mathrm{C}$ y $\beta C$ ) y las xantófilas (Lut, Zea y $\beta \mathrm{Cr}$ ) durante la digestión DG y DI in vitro de las bebidas de mango se presentan en el Cuadro 3. El contenido de $\alpha \mathrm{C}$ se incrementó en la DI, frente a la DG en la BC. Sin embargo, en esta misma bebida, el contenido de $\beta C$ presentó una disminución de compuestos. Estos resultados fueron similares a los encontrados en los zumos de la mezcla de frutas (kiwi, piña, mango, naranja) analizados por Rodríguez-Roque et al. (2014, 294). También pueden deberse al cambio de $\mathrm{pH}$ en las diferentes etapas de la digestión (RodríguezRoque et al., 2014, 294). Además, se sabe que la estructura del $\beta C$ no cambia durante la DI (Blanquet-Diot et al., 2009, 160). Por esta razón, el contenido de $\beta C$ permaneció similar entre la DG y en la DI. Al igual que el $\mathrm{BC}$, las xantófilas (Lut y $\beta \mathrm{Cr}$ ) permanecieron constantes durante el proceso de digestión en la BC (Cuadro 3). Sin embargo, el contenido de Zea se redujo entre ambas etapas en la BC. De tal manera, la liberación de carotenoides entre DG y DI depende de los componentes de la matriz del alimento, las interacciones de los carotenoides con otros componentes (fibra dietética, proteínas, ácidos grasos), la forma de preparación de bebidas, los cambios de $\mathrm{pH}$ entre las etapas de la digestión, entre otros (Estevez-Santiago et al., 2016, 438) son algunas de las razones por las cuales pueden existir estas diferencias. 
El Cuadro 3 se observa que al aplicar la EAU existe una disminución en el contenido de los carotenos y xantofilas en la DG y DI. El contenido de $\alpha \mathrm{C}, \beta \mathrm{C}, \beta \mathrm{Cr}$ disminuyó con respecto a $\mathrm{BC}$ en la DG. En este sentido, la disminución de estos compuestos fue mayor en la B-EAU3. Sin embargo, el contenido de Lut fue mayor en la B-EAU1 con respecto a BC y las B-EAU1 y BEAU2 tuvieron mayor contenido de Zea con respecto a BC en la DG (Cuadro 3). De la misma manera, en la DI se aprecian estos comportamientos. Por lo tanto, las amplitudes altas generaron un efecto negativo sobre el contenido de las xantofilas (Song et al., 2015, 233).

El contenido de carotenos y xantofilas liberados durante la digestión in vitro de las EAU presentaron cambios importantes. El Cuadro 3 se observa un cambio significativo en la liberación de los carotenos y xantofilas entre la DG y DI. El $\alpha \mathrm{C}$ tuvo una diferencia significativa $(\mathrm{p}<0.05)$ entre las bebidas excepto en la B-EAU1. Las xantofilas ( $\beta \mathrm{C}, \beta \mathrm{Cr}$ y Lut) mostraron diferencias significativas $(\mathrm{p}<0.05)$ en todas las bebidas con la EAU. Sin embargo, la Zea presentó diferencia en todas las bebidas. En el Cuadro 3, se muestra que en la B-EAU1 el contenido de $\alpha \mathrm{C}$ aumentó en la DI. Sin embargo, el contenido de Lut disminuyó en la misma bebida en esta etapa digestiva. En cambio, el contenido de Zea aumentó en la B-EAU1 en la DI. Esto puede ser explicado en parte considerando que durante la EAU el $\alpha \mathrm{Cr}$, la neoluteina, neoluteina monoester y neoluteina epóxido A y B sufren cambios estructurales produciendo la Zea (Cano y Ancos, 1994, 252). Del mismo modo, el contenido de $\beta \mathrm{C}$ aumentó en la DI en todas las bebidas con la EAU. Por lo tanto, los resultados muestran que los carotenos y las xantofilas son estables en TE prolongados y en AS bajas del EAU (Poojary et al., 2016, 584; Song et al., 2015, 220). Sin embargo, el proceso de cavitación en la B-EAU3 ocasionado por elevadas AS, produjo una disminución en el contenido de $\beta \mathrm{Cr}$ y Lut, posiblemente debido a las reacciones de isomerización y/o oxidación (Song et al., $2015,236)$.

Cuadro 3. Contenido de carotenoides liberados en la digestión gástrica (DG) y digestión intestinal (DI) en las bebidas de mango 'Ataulfo'.

\begin{tabular}{|c|c|c|c|c|c|}
\hline & \multicolumn{4}{|c|}{ Bebidas de mango (Mangifera indica L.) 'Ataulfo' $(\mu \mathrm{g} / 100 \mathrm{~mL})$} \\
\hline & & $\mathbf{B C}^{\mathbf{1}}$ & B-EAU1 & B-EAU2 & B-EAU3 \\
\hline \multirow{3}{*}{ DG } & irote & & & & \\
\hline & $\alpha \mathrm{C}$ & $1.59 \pm 0.04^{\mathrm{aB}}$ & $0.79 \pm 0.02^{\mathrm{bA}}$ & $0.79 \pm 0.02^{\mathrm{bA}}$ & $0.05 \pm 0.001^{\mathrm{cB}}$ \\
\hline & $\beta C$ & $2.65 \pm 0.05^{\mathrm{aA}}$ & $0.005 \pm 0.0001^{\mathrm{dB}}$ & $0.009 \pm 0.002^{\mathrm{bB}}$ & $0.006 \pm 0.001^{\mathrm{cB}}$ \\
\hline
\end{tabular}

Xantofilas 
Efecto de la extracción asistida por ultrasonido en la liberación y bioaccesibilidad in vitro de carotenoides, en bebidas elaboradas con mango (Mangifera indica L.) 'Ataulfo'
$\beta \mathrm{Cr}$
$3.74 \pm 0.07^{\mathrm{aA}}$
$0.41 \pm 0.008^{\mathrm{bA}}$
$0.41 \pm 0.008^{\mathrm{bA}}$
$0.08 \pm 0.001^{\mathrm{cB}}$
Lut
$0.48 \pm 0.02^{\mathrm{bA}}$
$2.09 \pm 0.10^{\mathrm{aA}}$
$0.44 \pm 0.02^{\mathrm{bB}}$
$0.02 \pm 0.001^{\mathrm{cB}}$
Zea
$0.11 \pm 0.003^{\mathrm{bA}}$
$0.23 \pm 0.007^{\mathrm{aB}}$
$0.23 \pm 0.007^{\mathrm{aA}}$
$0.01 \pm 0.0003^{\mathrm{cB}}$

DI Caroteno
$\alpha \mathrm{C}$
$2.04 \pm 0.06^{\mathrm{aA}}$
$0.83 \pm 0.02^{\mathrm{bA}}$
$0.23 \pm 0.007^{\mathrm{cB}}$
$0.28 \pm 0.008^{\mathrm{cA}}$
$\beta C$
$2.35 \pm 0.04^{\mathrm{aA}}$
$0.06 \pm 0.001^{\mathrm{bA}}$
$0.03 \pm 0.0006^{\mathrm{cA}}$
$0.01 \pm 0.0002^{\mathrm{dA}}$

Xantofilas
$\beta \mathrm{Cr}$
$3.51 \pm 0.07^{\mathrm{aA}}$
$0.14 \pm 0.003^{\mathrm{cB}}$
$0.21 \pm 0.004^{\mathrm{bB}}$
$0.12 \pm 0.0002^{\mathrm{dA}}$
Lut
$0.43 \pm 0.02^{\mathrm{cA}}$
$1.68 \pm 0.08^{\mathrm{aB}}$
$0.63 \pm 0.03^{\mathrm{bA}}$
$0.31 \pm 0.01^{\mathrm{dA}}$
Zea
$0.04 \pm 0.001^{\mathrm{bB}}$
$0.36 \pm 0.01^{\mathrm{aA}}$
$0.01 \pm 0.0003^{\mathrm{cB}}$
$0.03 \pm 0.009^{\mathrm{bA}}$

${ }^{1}: \mathrm{BC}$ : bebida sin tratamiento. B-EAU: bebida con extracción asistida por ultrasonido: ${ }^{1} \mathrm{X}_{\mathrm{TE}}: 30$ $\min , \mathrm{X}_{\mathrm{A}}: 30 \%, \mathrm{X}_{\mathrm{C}}: 0.8 .{ }^{2} \mathrm{X}_{\mathrm{TE}}: 15 \mathrm{~min}, \mathrm{X}_{\mathrm{A}}: 70 \%, \mathrm{X}_{\mathrm{C}}: 0.4 .{ }^{3} \mathrm{X}_{\mathrm{TE}}: 12 \min , \mathrm{X}_{\mathrm{A}}: 100 \%, \mathrm{X}_{\mathrm{C}}: 1 . \alpha-$ caroteno $(\alpha \mathrm{C}), \beta$-caroteno $(\beta \mathrm{C}), \beta$-criptoxantina $(\beta \mathrm{Cr})$, luteina (Lut) y zeaxantina (Zea). Los valores representan media \pm desviación estándar de tres repeticiones. Diferentes letras minúsculas representan diferencia significativa entre los diferentes tratamientos y el control. Diferentes letras mayúsculas representan diferencia significativa entre etapas de digestión.

Por otro lado, al hacer un comparativo entre el contenido de los carotenos y xantofilas sin el proceso de digestión in vitro (Cuadro 2) y con el proceso de digestión in vitro (Cuadro 3) se puede observar que el contenido de $\alpha \mathrm{C}, \beta \mathrm{C}, \beta \mathrm{Cr}$ y Zea en la $\mathrm{BC}$ aumenta en la DI. En contraste, en las B-EAUs los carotenos y las xantofilas dismininuyeron a excepción de la Zea ( $\mathrm{X}_{\mathrm{TE}}: 30 \mathrm{~min}, \mathrm{X}_{\mathrm{AS}}: 30 \%$, $\mathrm{X}_{\mathrm{C}}: 0.8$ ), $\beta C r\left(X_{\mathrm{TE}}: 12 \mathrm{~min}, \mathrm{X}_{\mathrm{AS}}: 70 \%, \mathrm{X}_{\mathrm{C}}: 0.4\right)$ y Zea $\left(\mathrm{X}_{\mathrm{TE}}: 12 \mathrm{~min}, \mathrm{X}_{\mathrm{AS}}: 100 \%, \mathrm{X}_{\mathrm{C}}: 1\right)$ (Cuadro 3). Por lo tanto, a pesar del incremento en Zea la EAU tuvo un impacto negativo sobre la bioaccesibilidad de los carotenoides cuando se encontraron en medios acuosos y sometidos a EAU (Carrillo-López et al., 2017, 52; Corte-Real et al., 2017; 14).

Cuadro 4. Contenido de carotenos y xantófilas asociadas a la fracción indigestible soluble (FIS) y fracción indigestible insoluble (FII) en las bebidas de mango 'Ataulfo'".

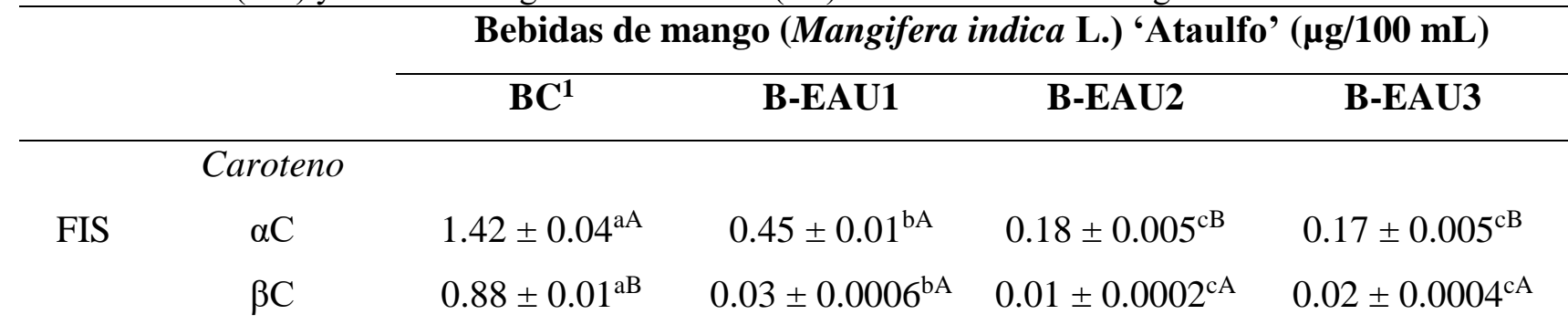

Xantofilas 


$\begin{array}{lcccc}\beta \mathrm{Cr} & 1.25 \pm 0.02^{\mathrm{aA}} & 0.04 \pm 0.0008^{\mathrm{cB}} & 0.08 \pm 0.001^{\mathrm{bB}} & 0.09 \pm 0.001^{\mathrm{bA}} \\ \text { Lut } & 0.40 \pm 0.02^{\mathrm{bA}} & 0.76 \pm 0.04^{\mathrm{aA}} & 0.39 \pm 0.02^{\mathrm{cA}} & 0.25 \pm 0.01^{\mathrm{dA}} \\ \text { Zea } & 0.02 \pm 0.0006^{\mathrm{cA}} & 0.15 \pm 0.004^{\mathrm{aA}} & 0.005 \pm 0.001^{\mathrm{dB}} & 0.03 \pm 0.0009^{\mathrm{bA}}\end{array}$

FII Caroteno
$\alpha \mathrm{C}$
$0.99 \pm 0.03^{\mathrm{aB}}$
$0.19 \pm 0.005^{\mathrm{cB}}$
$0.51 \pm 0.01^{\mathrm{bA}}$
$0.51 \pm 0.01^{\mathrm{bA}}$
$\beta C$
$0.99 \pm 0.02^{\mathrm{aA}}$
$0.01 \pm 0.0004^{\mathrm{bB}}$
$0.01 \pm 0.0004^{\mathrm{bA}}$
$0.009 \pm 0.0001^{\mathrm{cB}}$

Xantofilas
$\beta \mathrm{Cr}$
$1.58 \pm 0.03^{\mathrm{aA}}$
$0.09 \pm 0.002^{\mathrm{cA}}$
$0.10 \pm 0.002^{\mathrm{bA}}$
$0.10 \pm 0.002^{\mathrm{bA}}$
Lut
$0.34 \pm 0.01^{\mathrm{aA}}$
$0.20 \pm 0.01^{\mathrm{cB}}$
$0.29 \pm 0.01^{\mathrm{bB}}$
$0.29 \pm 0.01^{\mathrm{bA}}$
Zea
$0.02 \pm 0.0006^{\mathrm{cA}}$
$0.19 \pm 0.005^{\mathrm{aB}}$
$0.05 \pm 0.001^{\mathrm{bA}}$
$0.02 \pm 0.0006^{\mathrm{cA}}$

:BC: bebida sin tratamiento. B-EAU: bebida con extracción asistida por ultrasonido: ${ }^{1} \mathrm{X}_{\mathrm{TE}}$ : $30 \min , \mathrm{X}_{\mathrm{A}}: 30 \%, \mathrm{X}_{\mathrm{C}}: 0.8 .{ }^{2} \mathrm{X}_{\mathrm{TE}}: 15 \mathrm{~min}, \mathrm{X}_{\mathrm{A}}: 70 \%, \mathrm{X}_{\mathrm{C}}: 0.4 .{ }^{3} \mathrm{X}_{\mathrm{TE}}: 12 \mathrm{~min}, \mathrm{X}_{\mathrm{A}}: 100 \%, \mathrm{X}_{\mathrm{C}}: 1$. $\alpha$-caroteno $(\alpha \mathrm{C}), \beta$-caroteno ( $\beta \mathrm{C}), \beta$-criptoxantina $(\beta \mathrm{Cr})$, luteina (Lut) y zeaxantina (Zea). Los valores representan media \pm desviación estándar de tres repeticiones. Diferentes letras minúsculas representan diferencia significativa entre los diferentes tratamientos y el control. Diferentes letras mayúsculas representan diferencia significativa entre etapas de digestión.

En el Cuadro 4 se muestra el contenido de los carotenos y xantófilas que están asociados en la FIS y FII. El contenido de $\alpha \mathrm{C}$ de todas las bebidas presentó diferencia significativa $(\mathrm{p}<0.05)$ entre FIS y FII. En contraste, el $\beta C$ de la B-EAU no presentó diferencia significativa ( $p>0.05$ ). Sin embargo, todas las xantofilas de $\mathrm{BC}$ no presentaron diferencia significativa entre FIS y FII. Por el contrario, la $\beta \mathrm{Cr}$ y Lut en las B-EAU1 y B-EAU2 presentaron diferencia significativa entre FIS y FII (p $<0.05)$. En contaste; las tres xantofilas en la B-EAU3 no tuvieron diferencia significativa ( $p>0.05)$. La presencia de carotenoides en la FIS y FII se puede deber a que la interacción entre la fibra dietética (cáscara, pasta, pulpa) con la goma xantana mantiene a los carotenoides atrapados en una red que retarda o impide su liberación durante el proceso de digestión (Goñi et al., 2006, 236; Fernández-García et al., 2012, 91). Sin embargo, la energía que se generó durante el proceso de la cavitación facilitó la liberación de los carotenoides al romper las interacciones intermoleculares entre la goma xantana con la fibra dietética y los enlaces del complejo fibra-carotenoide (D'Evoli et al., 2013, 140; Palafox-Carlos et al., 2011, 268). En concordancia con los valores de carotenoides durante el proceso digestivo in vitro (Cuadro 3), el contenido de $\alpha \mathrm{C}, \beta \mathrm{C}$ y $\beta \mathrm{Cr}$ disminuyeron en los EAUs (Cuadro 3). Sin embargo, la presencia de los carotenos y las xantofilas en la FIS y FII puede deberse a que estos compuestos están asociados con otros componentes formando interacciones covalentes y puentes de hidrógeno que impiden su liberación (Palafox-Carlos et al., 2011, 268; 
D'Evoli et al., 2013, 142). De tal manera, su retención en la FIS y FII pueden llegar al intestino grueso y pueden tener un efecto favorable en el colon por la acción de la microbiota (Sen et al., 2013, 11; Taipina et al., 2013, 58). Cabe mencionar, que no existen estudios sobre el efecto positivo y/o negativo del contenido de carotenoides en la FII, lo que abre la posibilidad de estudiar el efecto que pueden ejercer los carotenoides en el colon.

\section{Efecto de la EAU sobre el porcentaje de bioaccesibilidad de los carotenoides}

En la Figura 1 se muestra el \%BA de carotenos $(\alpha \mathrm{C}, \beta \mathrm{C})$ (Figura 1A) y xantofilas (Lut, $\beta \mathrm{Cr}$ y Zea) (Figura 1B), en las bebidas de mango.

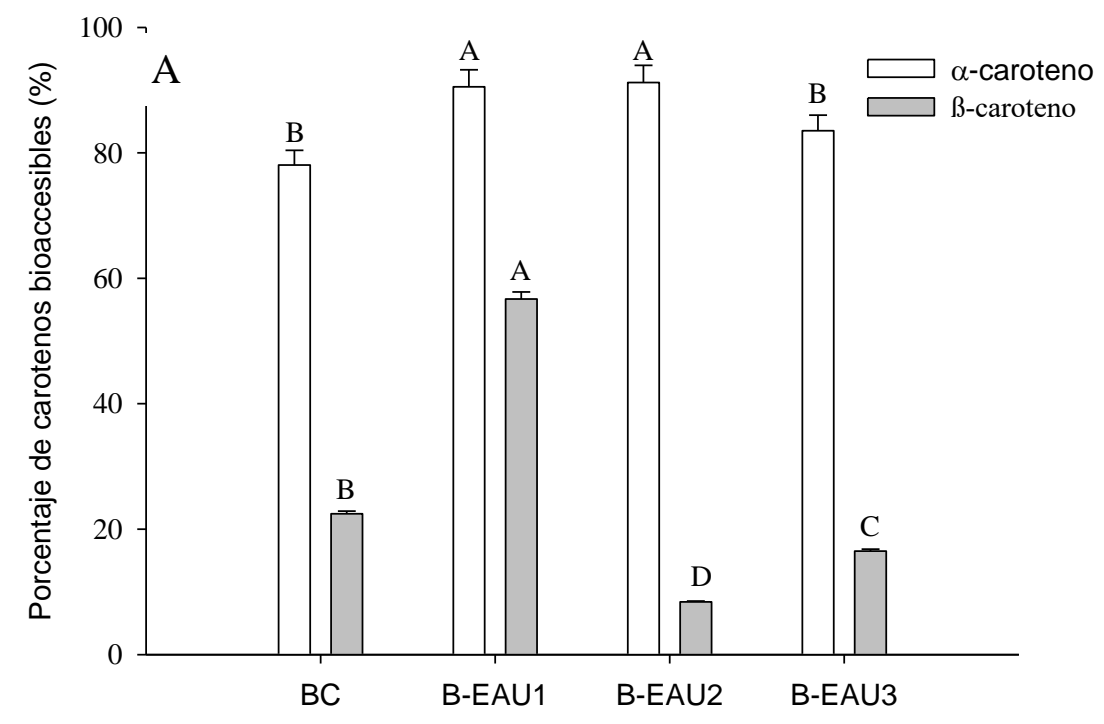




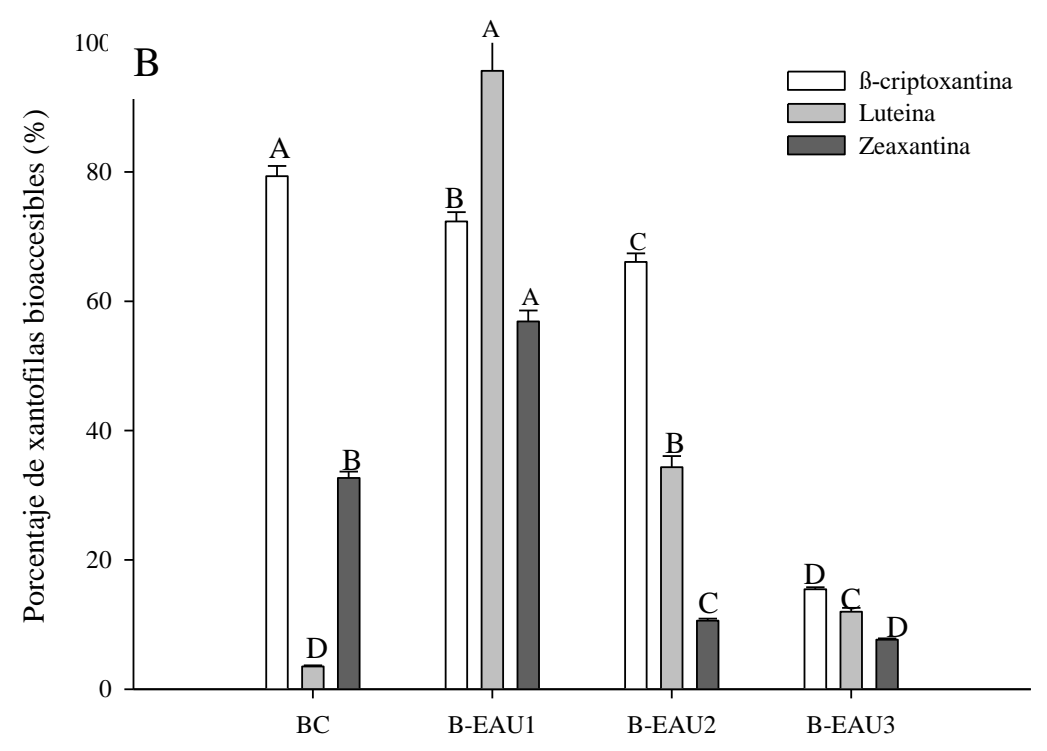

Figura 1. Porcentaje de bioaccesibilidad de (A) carotenos ( $\alpha$-C y $\beta$-C) y (B) xantófilas (Lut, Zea y $\beta$-Cr) en las bebidas de mango 'Ataulfo' (BC: bebida sin tratamiento. B-EAU: bebida con tratamiento de ultrasonido-asisitido: 1 ( $\mathrm{X}_{\mathrm{TE}}: 30 \mathrm{~min}, \mathrm{X}_{\mathrm{A}}: 30 \%, \mathrm{X}_{\mathrm{C}}:$ 0.8); 2( $\mathrm{X}_{\mathrm{TE}}: 15 \mathrm{~min}$, $\left.\mathrm{X}_{\mathrm{A}}: 70 \%, \mathrm{X}_{\mathrm{C}}: 0.4\right) ; 3\left(\mathrm{X}_{\mathrm{TE}}: 12 \mathrm{~min}, \mathrm{X}_{\mathrm{A}}: 100 \%, \mathrm{X}_{\mathrm{C}}: 1\right)$. Los valores representan media \pm desviación estándar de tres repeticiones. Diferentes letras representan diferencia significativa entre los diferentes tratamientos y el control.

La Figura 1A muestra que el $\alpha \mathrm{C}$ presentó un \%BA mayor en todos los EAU, respecto a la $\mathrm{BC}$, con un $91.22 \%$ (B-EAU2), $90.54 \%$ (B-EAU1) y $83.52 \%$ (B-EAU3). Por otra parte, el \%BA de $\beta C$ fue mayor en la B-EAU1 (56.68 \%). La Figura 1B muestra que la $\beta \mathrm{Cr}$ presentó un \%BA mayor $(79.35 \%)$ en $\mathrm{BC}$, en comparación con los tratamientos con EAU, por lo que la cavitación tuvo un efecto negativo en la estructura de esta xantofila. Sin embargo, para el resto de las xantofilas (Lut y Zea) el \%BA fue mayor en el tratamiento B-EAU1 con un 95.63 y $56.88 \%$, respectivamente. Por lo tanto, las amplitudes altas y los tiempos de exposición prolongados (B-EAU1) favorecen la libración de los carotenos ( $\alpha$ C y $\beta C$ ), Lut y Zea (Ofori-Boateng y Lee, 2013, 301). Por último, el $\beta C$ es el caroteno más abundante en el mango, el cual se puede encontrar en forma all-trans, allcis y cis-9 (Figura 1B) (Low et al., 2015, 70; Khoo et al., 2010, 8). De tal manera, la concentración de estos compuestos, las condiciones de las B-EAUs pueden contribuir en el \%BA de los carotenos.

Las xantofilas (Lut, Zea) tienen actividad provitamínica, previenen patologías crónicas como el cáncer, enfermedades cardiovasculares, enfermedades de la visión (cataratas y 
Efecto de la extracción asistida por ultrasonido en la liberación y bioaccesibilidad in vitro de carotenoides, en bebidas elaboradas con mango (Mangifera indica L.) 'Ataulfo'

degeneración macular asociada a la edad) y enfermedades relacionadas al envejecimiento (Taipina et al., 2013, 60; Edwards, 2016, 29; Mares, 2016, 6).

Por otra parte, se determinó el porcentaje de los carotenos y xantófilos no bioaccesibles a partir de la diferencia del \%BA (Figura 2).
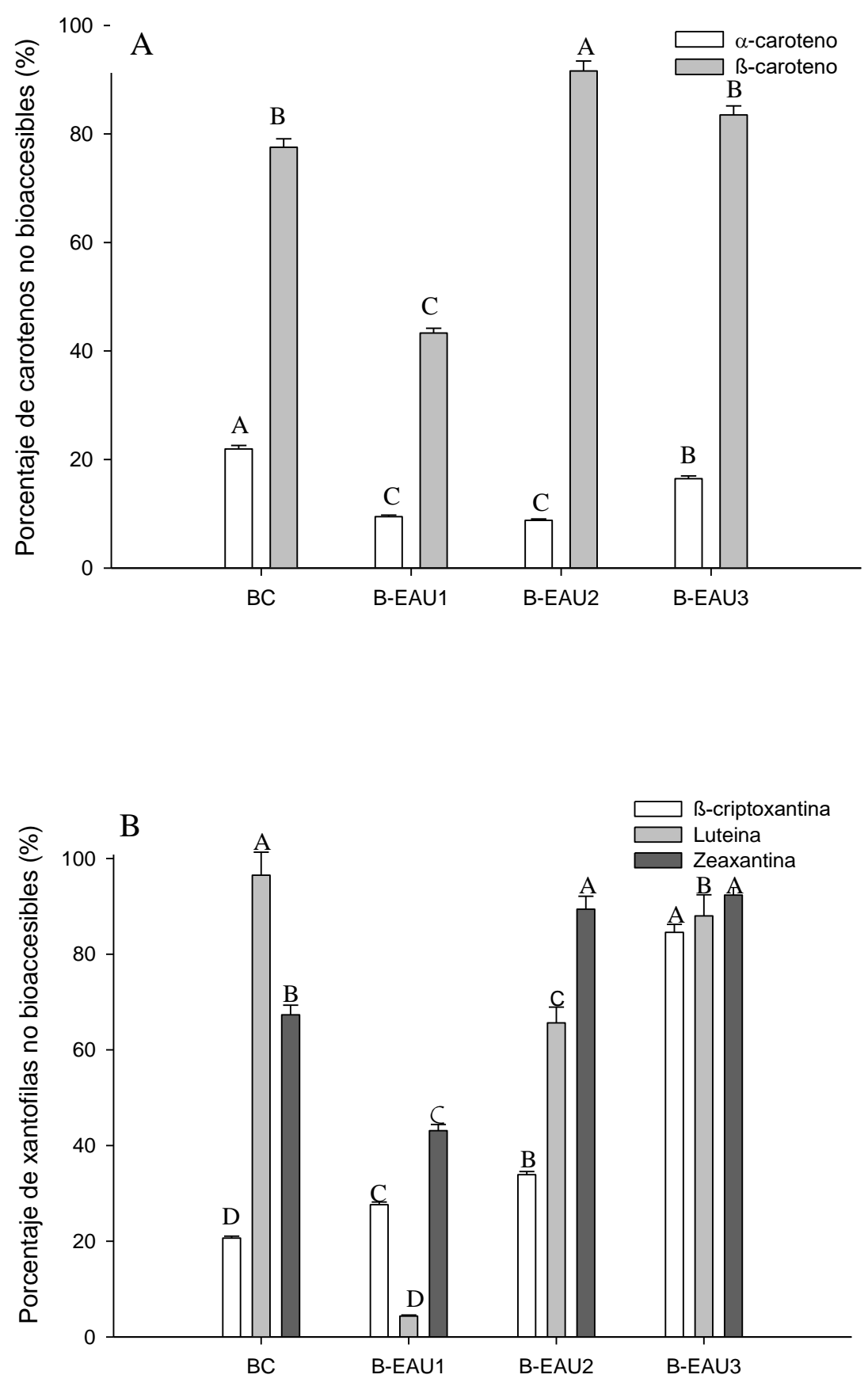
Figura 2. Porcentaje no bioaccesible de (A) carotenos $(\alpha-\mathrm{C}$ y $\beta$-C) y (B) xantofilas (Lut, Zea y $\beta$-Cr) en las bebidas de mango 'Ataulfo' (BC: bebida sin tratamiento. B-EAU: bebida con tratamiento de ultrasonido-asisitido: bebida con tratamiento de ultrasonido-asisitido: $1\left(\mathrm{X}_{\mathrm{TE}}\right.$ : $\left.30 \min , \mathrm{X}_{\mathrm{A}}: 30 \%, \mathrm{X}_{\mathrm{C}}: 0.8\right)$; 2(X $\left.\mathrm{X}_{\mathrm{TE}}: 15 \mathrm{~min}, \mathrm{X}_{\mathrm{A}}: 70 \%, \mathrm{X}_{\mathrm{C}}: 0.4\right) ; 3\left(\mathrm{X}_{\mathrm{TE}}: 12 \mathrm{~min}, \mathrm{X}_{\mathrm{A}}: 100 \%\right.$, $\left.\mathrm{X}_{\mathrm{C}}: 1\right)$. Los valores representan media \pm desviación estándar de tres repeticiones. Diferentes

letras representan diferencia significativa entre los diferentes tratamientos y el control.

Los carotenos no bioaccesibles son aquellos que quedaron retenidos en la FIS y la FII. El porcentaje de $\beta C$ no bioaccesible fue de 91.6, 83.5, 77.5 y 43.2 \% en las bebidas B-EAU2, B-EAU3, BC y BEAU1, respectivamente (Figura 2A). El porcentaje no bioaccesible del $\alpha \mathrm{C}$ fue mayor (21.9\%) en la $\mathrm{BC}$ respecto a las demás bebidas. También, en la misma bebida se observó el mismo efecto en la Lut (96.5\%). El mayor porcentaje de $\beta$ Cr y Zea no bioaccesibles se presentó en la BC y B-EAU3 con un 96.5 y $92.3 \%$, respectivamente (Figura 2B). Por lo tanto, encontrar xantofilas y carotenos asociados en la fracción indigestible de las bebidas de mango pueden ejercer funciones en el colon, ya que son inmunomodulares, por ejemplo, astaxantina, $\beta \mathrm{Cr}$ y Zea favorecen la inhibición del cáncer de colon (Kuppusamy et al., 2014, 146). Así mismo, el $\beta C$ puede favorecer la proliferación de microbiota precursora de la fermentación colónica. Se conoce que el $\beta C$ es un sustrato para el crecimiento de E. coli, el cual produce ácidos grasos de cadena corta (Jawed et al., 2016, 202; Wassef et al., 2014, 232).

\section{Cinética de liberación de los carotenoides}

El contenido de $\alpha \mathrm{C}, \beta \mathrm{C}, \beta \mathrm{Cr}$, Lut y Zea liberado de las bebidas de mango se muestra en el Cuadro 5 , donde se observa que la liberación de $\alpha \mathrm{C}$ fue mayor $(1.92 \mu \mathrm{g} / 100 \mathrm{~mL})$, seguido de $\beta \mathrm{Cr}(0.82$ $\mu \mathrm{g} / 100 \mathrm{~mL})$, Lut $(0.40 \mu \mathrm{g} / 100 \mathrm{~mL})$, Zea $(0.12 \mu \mathrm{g} / 100 \mathrm{~mL})$ y $\beta \mathrm{C}(0.08 \mu \mathrm{g} / 100 \mathrm{~mL})$ en la BC a los $180 \mathrm{~min}$. Este comportamiento se puede explicar considerando que existe una acción sinérgica o antagónica entre ellos durante su liberación (Ordoudi et al., 2015, 20; Fernández-García et al., 2008). También, durante el proceso de la cinética de liberación, existen otros compuestos que pudieran estar ejerciendo estas acciones sobre los carotenoides tales como la fibra dietética (Ordoudi et al., 2015, 22).

Al comparar la liberación de los carotenos y las xantofilas en las B-EAUs, se observó que presentaron mayor velocidad de liberación con respecto a la BC. En el Cuadro 5 se observa que los carotenos $(\alpha \mathrm{C}$ y $\beta \mathrm{C})$ en las B-EAUs no tuvieron diferencia significativa a los $180 \mathrm{~min}$. Sin embargo, la liberación de $\beta \operatorname{Cr}(9.15 \mu \mathrm{g} / 100 \mathrm{~mL})$ en la B-EAU1 fue mayor que el resto de las bebidas. Del mismo modo, Lut y Zea tuvieron mayor liberación en la B-EAU2. Por tal motivo, se considera que 
Efecto de la extracción asistida por ultrasonido en la liberación y bioaccesibilidad in vitro de carotenoides, en bebidas elaboradas con mango (Mangifera indica L.) 'Ataulfo'

el efecto de las condiciones del EAU influye en la liberación de estos compuestos (Goula et al., 2017, 254).

Cuadro 5. Cinética de liberación de $\alpha$-caroteno $(\alpha \mathrm{C}), \beta$-caroteno $(\beta \mathrm{C}), \beta$-criptoxantina $(\beta \mathrm{Cr})$, luteina (Lut), zeaxantina (Zea) en las bebidas de mango 'Ataulfo'.

\begin{tabular}{|c|c|c|c|c|c|}
\hline & \multirow[t]{2}{*}{ Tiempo (min) } & \multicolumn{4}{|c|}{ Bebidas de mango (Mangifera indica L.) 'Ataulfo' $(\mu \mathrm{g} / 100 \mathrm{~mL})$} \\
\hline & & $\mathbf{B C}^{\mathbf{1}}$ & B-EAU1 & B-EAU2 & B-EAU3 \\
\hline \multirow{6}{*}{$\alpha \mathrm{C}$} & 30 & $0.21 \pm 0.006^{\mathrm{b}}$ & $0.77 \pm 0.02^{\mathrm{a}}$ & $0.11 \pm 0.003^{\mathrm{c}}$ & $0.07 \pm 0.002^{\mathrm{d}}$ \\
\hline & 60 & $0.25 \pm 0.007^{\mathrm{b}}$ & $0.78 \pm 0.02^{\mathrm{a}}$ & $0.25 \pm 0.007^{\mathrm{b}}$ & $0.14 \pm 0.004^{\mathrm{c}}$ \\
\hline & 90 & $0.49 \pm 0.01^{\mathrm{c}}$ & $0.83 \pm 0.02^{\mathrm{a}}$ & $0.61 \pm 0.01^{\mathrm{b}}$ & $0.28 \pm 0.008^{\mathrm{d}}$ \\
\hline & 120 & $0.50 \pm 0.01^{\mathrm{c}}$ & $0.97 \pm 0.03^{\mathrm{a}}$ & $0.63 \pm 0.02^{b}$ & $0.40 \pm 0.01^{\mathrm{d}}$ \\
\hline & 150 & $0.62 \pm 0.02^{c}$ & $1.63 \pm 0.05^{\mathrm{a}}$ & $1.50 \pm 0.04^{\mathrm{a}}$ & $0.71 \pm 0.02^{\mathrm{b}}$ \\
\hline & 180 & $1.92 \pm 0.05^{\mathrm{b}}$ & $2.28 \pm 0.06^{\mathrm{a}}$ & $2.43 \pm 0.07^{\mathrm{a}}$ & $2.63 \pm 0.08^{a}$ \\
\hline \multirow{6}{*}{$\beta C$} & 30 & $0.02 \pm 0.0004^{b}$ & $0.11 \pm 0.002^{\mathrm{a}}$ & $0.004 \pm 0.001^{\mathrm{c}}$ & $0.11 \pm 0.002^{\mathrm{a}}$ \\
\hline & 60 & $0.04 \pm 0.008^{\mathrm{d}}$ & $0.11 \pm 0.002^{c}$ & $0.53 \pm 0.01^{\mathrm{a}}$ & $0.28 \pm 0.005^{\mathrm{b}}$ \\
\hline & 90 & $0.04 \pm 0.008^{\mathrm{d}}$ & $0.92 \pm 0.02^{\mathrm{b}}$ & $1.02 \pm 0.02^{\mathrm{a}}$ & $0.39 \pm 0.008^{c}$ \\
\hline & 120 & $0.04 \pm 0.008^{\mathrm{d}}$ & $1.63 \pm 0.03^{b}$ & $2.37 \pm 0.04^{\mathrm{a}}$ & $0.65 \pm 0.01^{\mathrm{c}}$ \\
\hline & 150 & $0.07 \pm 0.001^{\mathrm{c}}$ & $3.22 \pm 0.06^{\mathrm{a}}$ & $3.03 \pm 0.06^{\mathrm{a}}$ & $1.14 \pm 0.02^{b}$ \\
\hline & 180 & $0.08 \pm 0.001^{\mathrm{b}}$ & $4.84 \pm 0.09^{\mathrm{a}}$ & $4.30 \pm 0.08^{a}$ & $4.74 \pm 0.09^{a}$ \\
\hline \multirow{6}{*}{$\beta \mathrm{Cr}$} & 30 & $0.26 \pm 0.005$ & $2.88 \pm 0.05$ & $1.47 \pm 0.03$ & $0.48 \pm 0.009$ \\
\hline & 60 & $0.26 \pm 0.005$ & $3.54 \pm 0.07$ & $1.48 \pm 0.03$ & $0.73 \pm 0.01$ \\
\hline & 90 & $0.33 \pm 0.006$ & $4.96 \pm 0.09$ & $1.49 \pm 0.03$ & $0.80 \pm 0.01$ \\
\hline & 120 & $0.35 \pm 0.007$ & $6.43 \pm 0.12$ & $2.61 \pm 0.05$ & $1.02 \pm 0.02$ \\
\hline & 150 & $0.35 \pm 0.007$ & $7.49 \pm 0.15$ & $2.78 \pm 0.05$ & $2.53 \pm 0.05$ \\
\hline & 180 & $0.82 \pm 0.01$ & $9.15 \pm 0.18$ & $3.95 \pm 0.08$ & $6.16 \pm 0.12$ \\
\hline \multirow{6}{*}{ Lut } & 30 & $0.07 \pm 0.003^{c}$ & $0.49 \pm 0.02^{\mathrm{a}}$ & $0.45 \pm 0.02^{\mathrm{b}}$ & $0.001 \pm 0.0005^{\mathrm{d}}$ \\
\hline & 60 & $0.11 \pm 0.005^{\mathrm{a}}$ & $0.55 \pm 0.02^{\mathrm{a}}$ & $0.45 \pm 0.02^{\mathrm{b}}$ & $0.004 \pm 0.0002^{\mathrm{c}}$ \\
\hline & 90 & $0.21 \pm 0.01^{\mathrm{c}}$ & $0.65 \pm 0.03^{\mathrm{a}}$ & $0.56 \pm 0.03^{\mathrm{b}}$ & $0.68 \pm 0.03^{\mathrm{a}}$ \\
\hline & 120 & $0.40 \pm 0.02^{\mathrm{d}}$ & $0.77 \pm 0.03^{\mathrm{a}}$ & $0.57 \pm 0.03^{\mathrm{c}}$ & $0.73 \pm 0.03^{b}$ \\
\hline & 150 & $0.43 \pm 0.02^{\mathrm{d}}$ & $0.71 \pm 0.03^{b}$ & $0.62 \pm 0.03^{\mathrm{c}}$ & $1.03 \pm 0.05^{\mathrm{a}}$ \\
\hline & 180 & $0.40 \pm 0.02^{\mathrm{d}}$ & $0.86 \pm 0.04^{\mathrm{c}}$ & $1.23 \pm 0.06^{\mathrm{a}}$ & $1.24 \pm 0.06^{\mathrm{b}}$ \\
\hline
\end{tabular}




$\begin{array}{cccccc}30 & 0.009 \pm 0.0002^{\mathrm{d}} & 0.04 \pm 0.001^{\mathrm{a}} & 0.02 \pm 0.006^{\mathrm{c}} & 0.03 \pm 0.0009^{\mathrm{b}} \\ \text { Zea } & 60 & 0.03 \pm 0.0009^{\mathrm{b}} & 0.08 \pm 0.002^{\mathrm{a}} & 0.02 \pm 0.006^{\mathrm{c}} & 0.03 \pm 0.0009^{\mathrm{b}} \\ & 90 & 0.14 \pm 0.004^{\mathrm{a}} & 0.11 \pm 0.003^{\mathrm{a}} & 0.03 \pm 0.009^{\mathrm{b}} & 0.03 \pm 0.0009^{\mathrm{b}} \\ & 120 & 0.13 \pm 0.004^{\mathrm{a}} & 0.12 \pm 0.003^{\mathrm{a}} & 0.03 \pm 0.009^{\mathrm{c}} & 0.06 \pm 0.0002^{\mathrm{b}} \\ & 150 & 0.13 \pm 0.004^{\mathrm{b}} & 0.13 \pm 0.004^{\mathrm{b}} & 0.05 \pm 0.001^{\mathrm{c}} & 0.65 \pm 0.02^{\mathrm{a}} \\ & 180 & 0.12 \pm 0.003^{\mathrm{c}} & 0.14 \pm 0.004^{\mathrm{c}} & 0.72 \pm 0.02^{\mathrm{b}} & 1.74 \pm 0.05^{\mathrm{a}}\end{array}$

BC: bebida sin tratamiento. B-EAU: bebida con extracción asistida por ultrasonido: 1 ( $\mathrm{X}_{\mathrm{TE}}$ : 30 min, $\left.\mathrm{X}_{\mathrm{A}}: 30 \%, \mathrm{X}_{\mathrm{C}}: 0.8\right) ; 2\left(\mathrm{X}_{\mathrm{TE}}: 15 \mathrm{~min}, \mathrm{X}_{\mathrm{A}}: 70 \%, \mathrm{X}_{\mathrm{C}}: 0.4\right) ; 3\left(\mathrm{X}_{\mathrm{TE}}: 12 \mathrm{~min}, \mathrm{X}_{\mathrm{A}}: 100 \%\right.$, $\left.\mathrm{X}_{\mathrm{C}}: 1\right)$. Los valores representan media \pm desviación estándar de tres repeticiones. Diferentes letras minúsculas representan diferencia significativa entre los diferentes tratamientos y el control.

Por otra parte, al comparar el cuadro anterior con la bioaccesibilidad, se puede concluir que el efecto de la EAU durante el proceso de digestión favoreció la liberación de estos compuestos, sin embargo su bioaccesibilidad fue menor. Estos resultados sugieren que existen factores que influyen negativamente sobre la bioaccesibilidad, como la presencia de algunos minerales (cofactores divalentes), la fibra dietética, el tipo de lípidos presentes, que son liberados durante el proceso de digestión (Eriksen et al., 2017, 51; Corte Real et al. 2016, 35). Por esta razón, se analizó la velocidad de liberación de los carotenos y xantofilas.

En la Figura 3 se muestran las velocidades de liberación de $\alpha \mathrm{C}, \beta \mathrm{C}$, Lut, Zea y $\beta \mathrm{Cr}$ en las bebidas del mango. En la Figura 3A y 3B se observa que el comportamiento de la liberación de $\alpha \mathrm{C}$ y $\beta C$ fueron similares en todas las muestras, respectivamente. Sin embargo, $\alpha \mathrm{C}$ y $\beta C$ tuvieron una mayor velocidad de liberación en la B-EAU3 en los 180 min (Figura 3A). 
Efecto de la extracción asistida por ultrasonido en la liberación y bioaccesibilidad in vitro de carotenoides, en bebidas elaboradas con mango (Mangifera indica L.) 'Ataulfo'
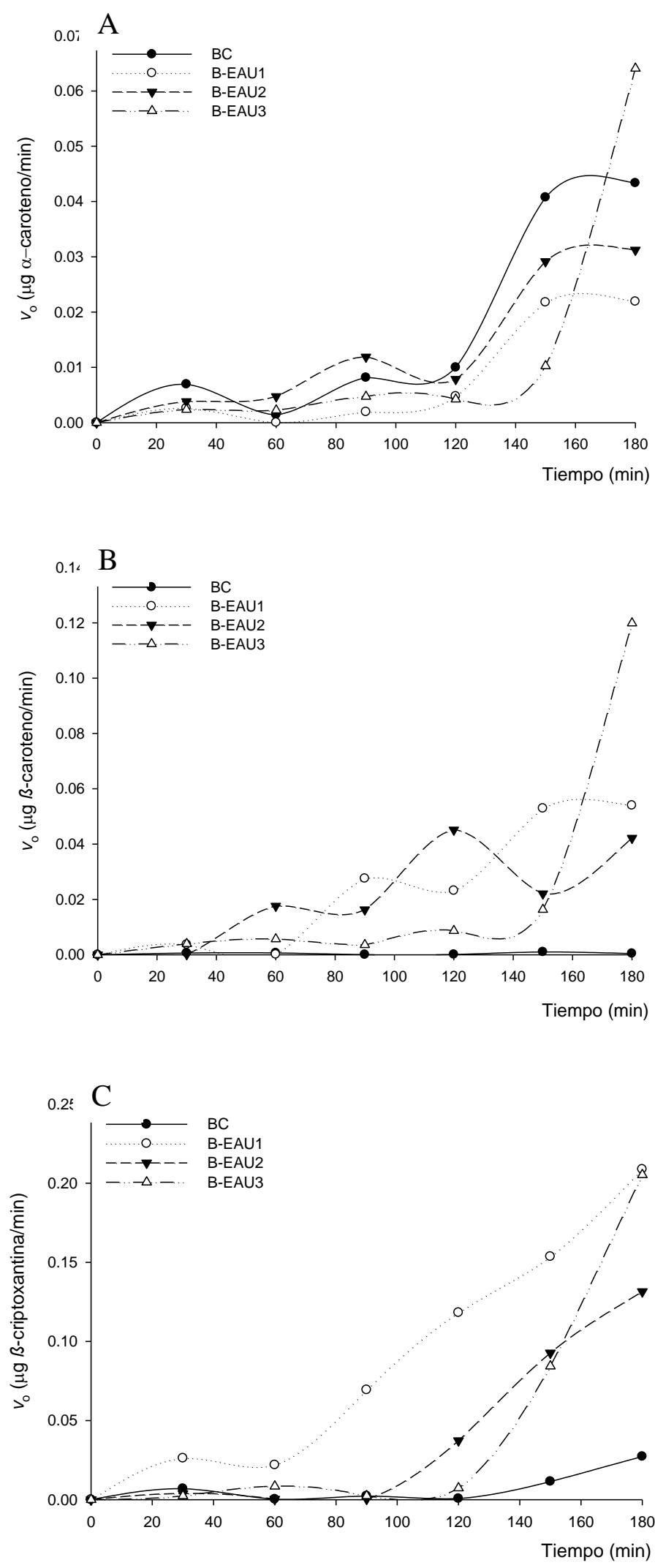

No 20, Vol. 10 (1), 2018. ISSN 2007 - 0705, pp.: 100 - 132 

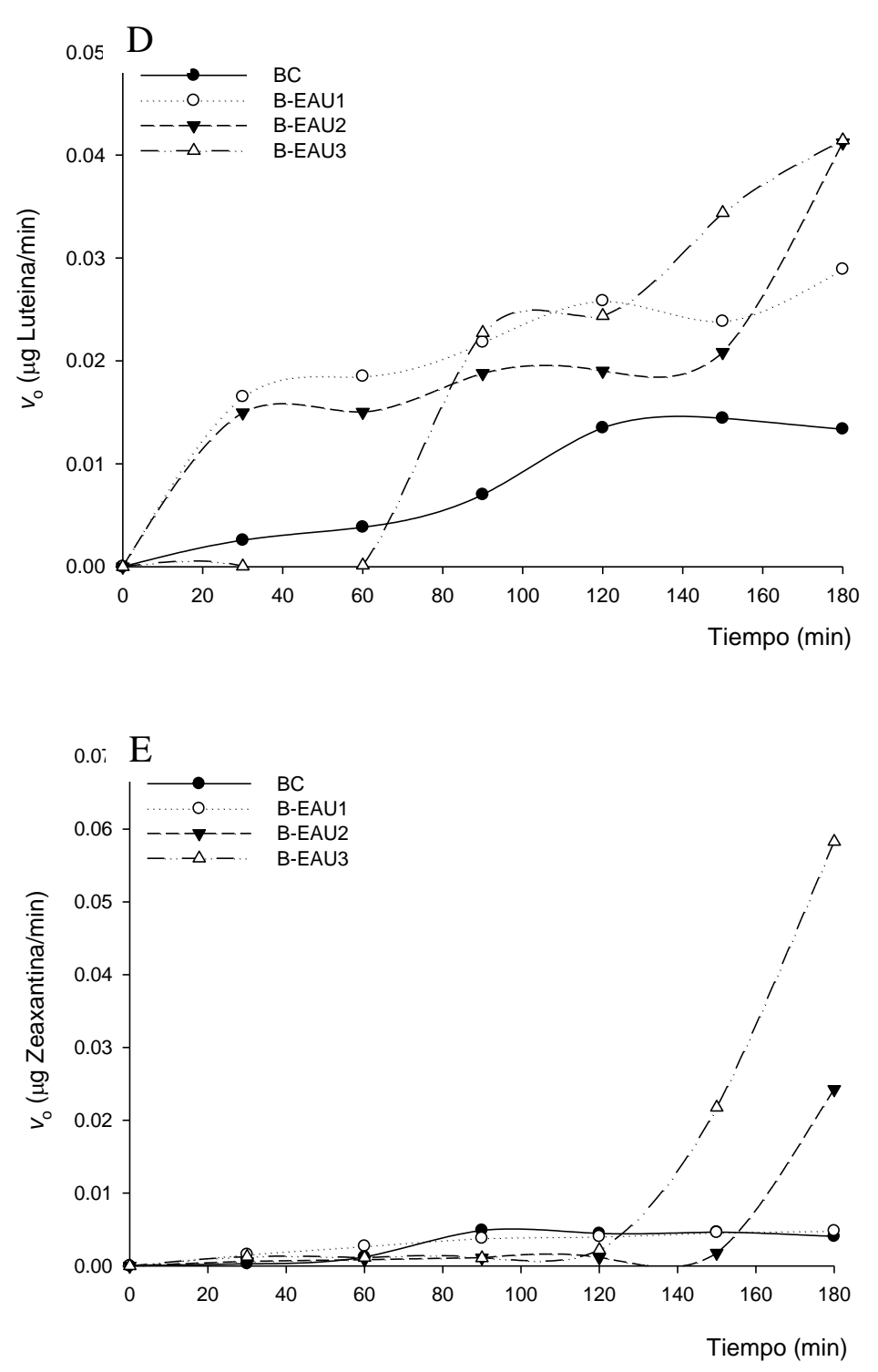

Figura 3. Velocidad de liberación de $\alpha$-Caroteno (A), $\beta$-Caroteno (B), $\beta$-Criptoxantina (C), Luteína (D) y Zeaxantina (E) en las bebidas de mango 'Ataulfo' (BC: bebida sin tratamiento.

B- EAU: bebida con tratamiento de ultrasonido-asistido: bebida con tratamiento de ultrasonido-asistido: $1\left(\mathrm{X}_{\mathrm{TE}}: 30 \mathrm{~min}, \mathrm{X}_{\mathrm{A}}: 30 \%, \mathrm{X}_{\mathrm{C}}: 0.8\right)$; $2\left(\mathrm{X}_{\mathrm{TE}}: 15 \mathrm{~min}, \mathrm{X}_{\mathrm{A}}: 70 \%, \mathrm{X}_{\mathrm{C}}: 0.4\right)$; $3\left(\mathrm{X}_{\mathrm{TE}}: 12 \mathrm{~min}, \mathrm{X}_{\mathrm{A}}: 100 \%, \mathrm{X}_{\mathrm{C}}: 1\right)$.

Las Figuras 3C y 3E muestran que la liberación de $\beta C r$ y Zea fueron a partir de los 120 min. En la Figura 3D se muestra la velocidad de liberación de Lut, en donde su liberación máxima estuvo en las muestras B-EAU2 y B-EAU3 a los 180 min. Estos resultados se deben que el $\beta C$, Zea y algunos ácidos grasos (ácido palmítico y laúrico) presentes en el mango facilitan el transporte por difusión facilitada de la Lut (Nwachukwu et al., 2016, 315; Nidhi et al., 2014, 257). Sin embargo, el efecto de la cavitación rompe los enlaces ésteres, puentes de hidrógeno y covalentes de las xantofilas con 
Efecto de la extracción asistida por ultrasonido en la liberación y bioaccesibilidad in vitro de carotenoides, en bebidas elaboradas con mango (Mangifera indica L.) 'Ataulfo'

la fibra dietética haciendo su liberación con mayor facilidad (Nwachukwu et al., 2016, 185; Altemimi et al., 2015, 103). Por lo tanto, se determinaron los parámetros cinéticos de la liberación de cada caroteno y xantofilas. En el Cuadro 6 se muestran los parámetros cinéticos $\mathrm{V} f$ y $k$, de los carotenos y xantófilas a partir de la cinética de liberación.

Cuadro 6. Determinación de los parámetros cinéticos del $\alpha$-caroteno $(\alpha \mathrm{C}), \beta$-caroteno $(\beta \mathrm{C})$, $\beta$-criptoxantina ( $\beta \mathrm{Cr}$ ), luteina (Lut) y zeaxantina (Zea) en las bebidas de mango 'Ataulfo' .

\section{Bebidas de mango (Mangifera indica L.) 'Ataulfo' $(\mu \mathrm{g} / 100 \mathrm{~mL}$ )}

\begin{tabular}{|c|c|c|c|c|}
\hline & $\mathrm{BC}^{\mathbf{1}}$ & B-EAU1 & B- EAU2 & B-EAU3 \\
\hline \multicolumn{5}{|l|}{$\alpha \mathrm{C}$} \\
\hline $\mathrm{V} f$ & 1.19 & 0.004 & 0.038 & 2.42 \\
\hline$k$ & 1.013 & 0.007 & 0.020 & 0.022 \\
\hline $\mathrm{R}$ & 0.8865 & 0.8641 & 0.8263 & 0.7974 \\
\hline \multicolumn{5}{|l|}{$\beta C$} \\
\hline $\mathrm{V} f$ & 2.007 & 2.007 & 2.53 & 2.41 \\
\hline$k$ & 0.009 & 0.028 & 0.039 & 0.022 \\
\hline $\mathrm{R}$ & 0.9505 & 0.8764 & 0.7276 & 0.9276 \\
\hline \multicolumn{5}{|l|}{$\beta \mathrm{Cr}$} \\
\hline $\mathrm{V} f$ & 2.44 & 0.004 & 3.76 & 2.422 \\
\hline$k$ & 0.006 & 0.008 & 0.007 & 0.016 \\
\hline $\mathrm{R}$ & 0.8809 & 0.9556 & 0.9363 & 0.8661 \\
\hline \multicolumn{5}{|l|}{ Lut } \\
\hline $\mathrm{V} f$ & 1.33 & 0.004 & 3.21 & 2.81 \\
\hline$K$ & 0.012 & 0.004 & 0.006 & 0.049 \\
\hline $\mathrm{R}$ & 0.9642 & 0.9041 & 0.9503 & 0.933 \\
\hline \multicolumn{5}{|l|}{ Zea } \\
\hline $\mathrm{V} f$ & 3.47 & 0.001 & 2.43 & 1.35 \\
\hline$k$ & 0.016 & 0.007 & 0.020 & 0.027 \\
\hline $\mathrm{R}$ & 0.9994 & 0.919 & 0.8674 & 0.8519 \\
\hline
\end{tabular}

:BC: bebida sin tratamiento. B-EAU: bebida con tratamiento de ultrasonido-asisitido: ${ }^{2} \mathrm{X}_{\mathrm{TE}}$ : $30 \min , \mathrm{X}_{\mathrm{A}}: 30 \%, \mathrm{X}_{\mathrm{C}}: 0.8 .{ }^{3} \mathrm{X}_{\mathrm{TE}}: 15 \mathrm{~min}, \mathrm{X}_{\mathrm{A}}: 70 \%, \mathrm{X}_{\mathrm{C}}: 0.4 .{ }^{4} \mathrm{X}_{\mathrm{TE}}: 12 \mathrm{~min}, \mathrm{X}_{\mathrm{A}}: 100 \%, \mathrm{X}_{\mathrm{C}}: 1$. $\mathrm{V} f$ : Velocidad final de liberación $(\mu \mathrm{g} / \mathrm{min}) ; k$ : constante cinética. 
En el Cuadro 6 se observan los parámetros estimados para los carotenos y las xantofilas en cada bebida. La Zea presentó una mayor liberación (3.47 $\mu \mathrm{g} / \mathrm{min})$ en BC. Sin embargo, en las bebidas con la EAU, el $\beta \mathrm{C}$ presentó una concentración mayor $(2.007 \mu \mathrm{g} / \mathrm{min})$ en $\mathrm{B}-\mathrm{EAU} 1, \beta \mathrm{Cr}(3.76$ $\mu \mathrm{g} / \mathrm{min})$ en B-EAU2 y Lut $(2.81 \mu \mathrm{g} / \mathrm{min})$ en la B- EAU3. También, en este Cuadro 6 se muestra el ajuste de los datos al modelo cinético de primer orden $(\mathrm{R})$, que ratifica que el proceso de liberación de los carotenos y xantofilas está controlado por reacciones químicas que ocurren durante el proceso de digestión y no por difusión. Por lo tanto, la variabilidad del ajuste de cada compuesto depende de la heterogeneidad del alimento lo cual es habitual cuando se trabaja con alimentos, en los que la composición no es únicamente dependiente del compuesto sino también de la cantidad de la muestra dentro del alimento (Shannon et al., 2017, 465; Kodal y Aksu, 2017, 130). Así mismo, la liberación de los carotenos y xantofilas va a depender de las propiedades de incremento de viscosidad que puede presentar la fibra dietética soluble lo que favorece un proceso de difusión más lento (Palafox-Carlos et al., 2011, 401; Kotake-Nara y Nagao, 2011, 33). De esta manera, estudios previos han demostrado que la matriz alimentaria, las interacciones con la fibra dietética, proteínas y ácidos grasos (esterificados o en forma libre), dentro de las micelas, pH del medio (en comparación con el agua), la competencia entre los mismos carotenoides, la formulación de ciertas bebidas, los tratamientos físicos y tecnológicos influyen en el comportamiento de la liberación de los compuestos de interés (Kong et al., 2016. 168; Domínguez-Cañedo et al., 2015, 425; Mardai et al., 2012, 201).

\section{Conclusiones}

Los SP de mango ‘Ataulfo' fueron utilizados en este estudio para elaborar bebidas con la aplicación del EAU. También, el uso de nuevas tecnologías como la EAU pueden resultar una alternativa viable para la extracción de estos compuestos y tener más bioaccesibles a los carotenos y xantofila. De esta manera, el contenido de las xantofilas y los carotenos de las bebidas dependió de cada condición de EAU. Sin embargo, la B-EAU1 tuvo la mayor bioaccesibilidad in vitro de luteína, zeaxantina, $\alpha$-caroteno y $\beta$-caroteno en un $95.63,56.88,58.68$, y $90.54 \%$, respectivamente. Sin embargo, la BC y la B-EAU3 tuvieron mayor retención a la $\beta$-criptoxantina (96.5 \%) y zeaxantina $(92.3 \%)$, respectivamente. Del mismo modo, los factores intrínsecos de las bebidas al igual que EAU influyeron en la velocidad de liberación de los carotenoides. 
Efecto de la extracción asistida por ultrasonido en la liberación y bioaccesibilidad in vitro de carotenoides, en bebidas elaboradas con mango (Mangifera indica L.) 'Ataulfo'

\section{$\underline{\text { Agradecimientos }}$}

GMM agradece al Consejo Nacional de Ciencia y Tecnología (CONACyT), por la beca de doctorado otorgada, número 241180. También se agradece al M. en C. Orlando Tortoledo-Ortíz y al Dr. Humberto Astiazarán-García por la capacitación de extracción de equinenona por el método de estándar externo para determinar el porcentaje de recuperación en el Laboratorio de Nutrición en el Centro de Investigación en Alimentación y Desarrollo A.C., Hermosillo.

\section{$\underline{\text { Referencias }}$}

Achoff, J. K., Kaufmann, S., Kalkan, O., Nedhart, S., Carle, R. y Schweiggert, R. M. (2014). In vitro bioccessibility of carotenoids, flavonoids, and vitamin $\mathrm{C}$ from differently processed oranges and orange juices [Citrus sinensis (L.) Osbeck]. Journal of Agricultural and Food Chemistry. 63: 578-587.

Altemimi, A., Lightfoot, D. A., Kinsel, M. y Watson, D. G. (2015). Employing response surface methodology for the optimization of ultrasound assisted extraction of lutein and $\beta$-carotene from spinach. Molecules. 20: 6611-6625.

Ajila, C M., Bhat, S. G. y Prasada-Rao, U. J. S. (2007). Valuable components of raw and ripe pells from two Indian mango varieties. Food Chemistry. 102: 1006-1011.

Blancas-Benítez, F. J., Avena-Bustillos, R. J., Montalvo-Gonzalez, E., Sáyago-Ayerdi, S. G. y McHugh, T. H. (2015a). Addition of dried 'Ataulfo' mango (Mangifera indica L) byproducts as a source of dietary fiber and polyphenols in starch molded mango snacks. Journal of Food Science and Technology. 52(11): 7393-7400.

Blancas-Benítez, F.J., Mercado-Mercado, G., Quirós-Sauceda, A.E., Montalvo-González, E., González-Aguilar, G.A. y Sáyago-Ayerdi, S.G. (2015b). Bioaccessibility of polyphenols associated with dietary fiber and in vitro kinetics reléase of polyphenols in Mexican 'Ataulfo' mango (Mangifera indica L.) by-products. Food and Function. 6(3): 859-868.

Blanquet-Diot, S., Soufi, M., Rambeau, M., Rock, E. y Alric, M. (2009). Digestive stability of xanthophylls esceeds that of carotenes as studied in a dynamic in vitro gastrointestinal system. Journal of Nutrition. 876-883.

Cano, M. P. y de Ancos. (1994). Carotenoid and carotenoid ester composition in mango fruit as influenced by processing method. Journal of Agricultural and Food Chemistry. 42(12): 2737-2742. 
Carail, M., Fabiano-Tixier, A. S., Meullemiestre, A., Chemat, F. \& Caris-Veyrat, C. (2015). Effects of high power ultrasound on all-E- $\beta$-carotene, newly formed compounds analysis by ultrahigh-performance liquid chromatography-tandem mass spectrometry. Ultrasonics Sonochemistry. 26: 200-209.

Carrillo-López, L. M., Alarcon-Rojo, A. D., Luna-Rodríguez, L. y Reyes-Villagrana, R. (2017). Modification of food system by ultrasound. Hindawi Journal of Food Quality. 12.

Chen, J. P., Tai, C. Y. y Chen, B. H. (2004). Improve liquid chromatographis method for determination of carotenoids in Taiwanese mango (Mangifera indica L.).Journal of Chrmatography A. 1054: 261-268.

Cilla, A., Alegría, A., de Ancos, B., Sánchez-Moreno, C., Pilar-Cano, M., Plaza, L., Clemente, G., Lagarda, M. J. y Barberá, R. (2012). Bioaccessibility of tocopherols, carotenoids, and ascorbic acid from milk- and soy-based fruit beverages: influence of food matrix and processing. Journal of Agricultural and Food Chemistry. 60: 7282-7290.

Colchero, M. A., Popkin, B. M., Rivera, J. A. y Ng, S. W. (2016). Beverage purchases from stores in Mexico under the excise tax on sugar sweetened beverages: observational study. British Medican Journal. 6: 352.

Corte-Real, J., Bertucci, M., Soukoulis, C., Desmarchelier, C., Borel, P., Richling, E., Hoffmann, L. y Bohn, T. (2017). Negeative effects of divalent mineral cations on the bioaccessibility of carotenoids from plant food matrices and related physical properties of gastro-intestinal fluids. Food and Function. 8: 1008-1019.

D’Evoli, L., Lombardi-Boccia, G. y Lucarini, M. (2013). Influence of heat treatments on carotenoid content of cherry tomatoes. Foods. 2: 352-363.

Domínguez-Cañedo, I. L., Beristain-Guevara, C. I., Díaz-Sobac, R. y Vázquez-Luna, A. (2015). Degradación de carotenoides y capsaicina en el complejo de inclusión molecular de oleorresina de chile habanero (Capsicum chinense) con $\beta$-ciclodextrina. CyTA - Journal of Food. 13(1): 151-158.

Edwards, J. A. (2016). Zeaxanthin, review of toxicological data and acceptable daily intake. Journal of Ophthalmology. 4: 1-15.

Eriksen, J. N., Luu, A. Y., Dragsted, L. O. y Arrigoni, E. (2017). Adaption of an in vitro digestión method tos creen carotenoid liberation and in vitro accessibility from differently processed spinach preparation. Food Chemistry. 224: 407-413. 
Estévez-Santiago, R., Olmedilla-Alonso, B. y Fernández-Jalao, I. (2016). Bioaccessibility of provitamin A carotenoids from fruits: application of a standardised static in vitro digestion method. Food and Fuction. 7: 1354-1366.

Fernández-García, E., Rincón, F. y Pérez-Gálvez, A. (2008). Developing an emulsifier system to improve the bioaccessibility of carotenoids. Journal of Agricultural and Food Chemistry. 56: 10384-10390.

Fernández-García, E., Carvajal-Lérida, I., Jarén-Galán, M., Garrido-Fernández, J., Pérez-Gálvez, A. y Hornero-Méndez, D. (2012). Carotenoids bioavailability from foods: from plant pigments to efficient biological activities. Food Research International. 46: 438-450.

Goula, A. M., Ververi, M., Adamopoulou, A. y Kaderides, K. (2017). Green ultrasound-assisted extraction of carotenoids from pomegrate wastes using vegetable oils. Ultrasonics Sonochemistry. 34: 821-830.

Fiedor, J. y Burda, K. (2014). Review Potential role of carotenoids as antioxidants in human health and disease. Nutrients. 6: 466-488.

Goñi, I., Serrano, J. y Saura-Calixto, F. (2006). Bioaccessibility of $\beta$-carotene, lutein, and lycopene from fruits and vegetables. Journal of Agricultural and Food Chemistry. 54: 5382-5387.

Janiszewska, E. y Sakowski, P. (2013). Effect of the ultrasound on the carrot juices freezing process. Inzynieria Rolnicza. 4(148): 49-57.

Izli, N., Izli, G. y Taskin, O. (2017). Influence of different drying techniques on drying parameters of mango. Food Science and Technology. 37(4): 604-612.

Jawed, K., Mattam, A. J., Fatma, Z., Wajid, S., Abdin, M. y Yazdani, S. S. (2016). Engineered production of short chain fatty acid in Escherichia coli using fatty acid synthesis pathway. PLOSone. 1-20.

Kiokas, S., Proestos, C. y Varzakas, T. (2016). A review of the structure, bosynthesis, absorption of carotenoids-analysis and properties of their common natural extracts. Current Research in Nutrition and Food Science. 4(Special Issue 1): 25-37.

Khoo, H. E., Nagendra-Praad, K., Kong, K. W., Jiang, Y. y Ismail, A. (2011). Review carotenoids and their isomers: color pigments in fruits and vegetables. Molecules. 16: 1710-1738.

Khoo, H. E., Nagendra-Prasad, K., Ismail, A. y Mohd-Esa, N. (2010). Carotenoids from Mangifera pajang and their antioxidant capacity. Molecules. 15: 6699-6712. 
Kodal, S. P. y Aksu, Z. (2017). Phenolic pigment extraction from orange peels: kinetic modeling. $15^{\text {th }}$ International Conference on Environmental Science and Technology. Rhodes, Greece. 31 August to 2 September 2017. 798-803.

Kong, F., Yu, S., Bi, Y., Huang, X. y Huang, M. (2016). Optimization of process parameters and kinetic model of enzymatic extraction of polyphenols from Lonicerae Flos. Pharmacognosy Magazine. 12(45): 70-74.

Kotake-Nara E. y Nagao, A. (2011). Absorption and metabolism of xanthophylls. Marine Drugs. 9: 1024-1037.

Kuppusamy, P., Yusoff, M. M., Maniam, G. P., Arief-Ichwan, S. J., Soundharrajan, I. y Govindan, N. (2014). Nutraceuticals as potential therapeutic agents for colon cancerL a review. Acta Pharmaceutica Sinica B. 4(3): 173-181.

Lauricella, M., Emanuele, S., Calvaruso, G., Giuliano, M. y D’Anneo, A. (2017). Multifaceted health benefits of Mangifera indica L. (Mango): the inestimable value of orchards recently planted in Sicilian rural areas. Nutrients. 9: 525.

Lin, K. H., Lin, K. C., Lu, W. J., Thomas, P. A., Jayakumar, T. y Sheu, J. R. (2016). Astaxanthin, a carotenoid, stimulates immune response by enhancing IFN- $\gamma$ all IL-2 secretion in primary cultured lymphocytes in vitro and ex vivo. International Journal of Molecular Science. 17: 44.

Low, D. Y., D’Arcy, B. y Gidley, M. J. (2015). Mastication effects on carotenoid bioaccessibility from mango fruit tissue. Food Research International. 67: 238-246.

Mares, J. (2016). Lutein and zeaxanthin isomers in eye health and disease. Reviews in Advance. Annual Reviews of Nutrition. 36: 24-29.

Mardai, A., Maldonado, F., Cuadros, S., Bautista, (2012). Adsoprtion, isotherm, thermodynamic and kinetcs studies of polyphenols onto tannery shavings. Chemical Engineering Journal. 185(15): 21-29.

Marx, M., Stuparic, M., Schieber, A. y Carle, R. (2003). Effect of thermal processing on trans-cisisomerization of $\beta$-carotene in carrot juices ad carotene-containing preparation. Food Chemistry. 83: 609-617.

Mercadante, A. Z., Rodríguez-Amaya, D. B. y Britton, G. (1998). HPLC and mass spectrometric analysis of carotenoids from mango. Journal of Agricultural and Food Chemistry. 45: 120123.

No 20, Vol. 10 (1), 2018. ISSN 2007 - 0705, pp.: 100 - 132 
Naldal, U. y Bhardwaj, R. L. (2012). Aloe vera for human nutrition, health and cosmetic use - A review. International Reearch Journal of Plant Science. 3(3): 38-46.

Nidhi, B., Ramaprasad, T. R. y Baskaran, V. (2014). Dietry fatty acid determines the intestinal absorption of lutein in lutein deficient mice. Food Research International. 64: 256-263.

Nwachukwu, I. D., Udenigwe, C. C. y Aluko, R. E. (2016). Review: lutein and zeaxanthin: production technology, bioavailability, mechanism of action, visual function, and health claim status. Trends in Food Science and Technology. 49: 74-84.

Ofori-Boateng, C. y Lee, K.T. (2013). Response surface optimization of ultrasonic-assisted extraction of carotenoids from oil palm (Elaeis guineensis Jacq.) fronds. Food Science and Nutrition. 1(3): 209-221.

Ordoudi, S. A., Kyriakoudi, A. y Tsimidou, M. Z. (2015). Enhanced bioaccessibility of crocetin sugar esters from saffron in infusions rich in natural phenolic antioxidants. Molecules. 20: 17760-17774.

Ornelas-Paz, J. J., Yahia, E. M. y Gardea, A. A. (2008). Changes in external and internal color during posthaervest ripening of 'Manila' and 'Ataulfo' mango fruit and relationship with carotenoid content determined by liquid chromatography-APcL+-time-of-flight mass spectrometry. Postharbest Biology and Technology. 50: 145-152.

Palafox-Carlos, H., Ayala-Zavala, J. F. y González-Aguilar, G. A. (2011). The role of dietary fiber in the bioaccessibility and bioavailability of fruit and vegetable antioxidants. Journal of Food Science. 76(1): R6-R15.

Periago, M. J., Martínez-Valverde, I., Ros, G., Martínez, C. y López, G. (2001). Propiedades químicas, biológicas y valor nutritivo del licopeno. Anales de Veterinaria de Murcia. 17: 51-66.

Petry, C. F. y Mercadante, A. Z. (2018). Short report New method for carotenoid extraction and analysis by HPLC-DAD-MS/MS in freeze-dried citrus and mango pulp. Journal of Brazilian Chemical Society. 29(1): 205-216.

Plaza, L., Crespo, I., de Pascual-Teresa, S., de Ancos, B., Sánchez-Moreno, C., Muñoz, M. y Pilar-Cano, M. (2011). Impact of minimal processing on orange bioactive compounds during refrigerated storage. Food Chemistry. 124: 646-651. 
Poojary, M. M., Barba, F. J., Aliakbarian, B., Donsi, F., Pataro, G., Dias, D. A. y Juliano, P. (2016). Innovative alternative technologies to extract carotenoids from microalgae and seaweeds. Marine Drugs. 14: 2-34.

Pott, I., Breithaupt, D. E. y Carle, R. (2003). Detection of unusual carotenoid ester in fresh mango (Mangifera indica L. cv. 'Kent'). Phytochemistry. 64: 825-829.

Qin, J., Yeum, K. J., Johnson, E., Krinsky, N. I., Russell, R. M. y Tang, G. (2008). Determination of 9 -cis $\beta$-carotene and $\zeta$-carotene in biological samples. Journal of Nutritional Biochemistry. 19: 612-618.

Radi, R., Firouzi, E., Akhavan, H. y Amiri, S. (2017). Effect of gelatin0based edible coating incorporated with Aloe vera and black and Green tea extracts on the shelf life of fresh-cut oranges. Hindawi Journal of Food Quality. 10.

Ravani, A. y Joshi, D. C. (2013). Mango and its by-product utilization - a review. Trend in Post Harvest Technology. 1(1): 55-67.

Rodríguez-Roque, M. J., Rojas-Graii, M. A., Elez-Martínez, P. y Martín-Belloso, O. (2013). Changes in vitamin $\mathrm{C}$, phenolic, and carotenoid profiles throughout in vitro gastrointestinal digesion of a blended fruit juice. Journal of Agricultural and Food Chemistry. 61: 18591867.

Rodríguez-Roque, M. J., Rojas-Graü, M. A., Martínez, P. E. y Martín-Belloso, O. (2014). In vitro bioaccessibility of health-related compounds as affected by the formulation of fruit juiceand milk-based beverages. Food Research International. 62: 771-778.

Saura-Calixto, F., García-Alonso, A. Goñi, I. y Bravo, L. (2000). In vitro determination of indigestible fraction in foods: an alternative to dietary fiber analysis. Journal of Agriculture and Food Chemistry. 48(8): 3342-3347.

Sen, A., Ren, J., Ruffin, M. T., Tergeon, D. K., Brenner, D. E., Sidahmed, E., Rapai, M. E., Cornellier, M. L. y Djuric, Z. (2013). Relationship between serum and colon concentrations of carotenoids and fatty acids in randomized dietary intervention trial. Cancer Prevention Research. 6(6): 558-565.

Shannon, E., Jaiswal, A. K. y Abu-Ghannam, N. (2017). Polyphenolic content and antioxidant capacity of white, green, black, and herbal teas: a kinetic study. Food Researh. 2(1): 1-11.

Song, J. F., Li, D. J., Pang, H. L. y Liu, C. Q. (2015). Effect of ultrasonic waves on the stability of all-trans lutein and its degradation kinetics. Ultrasonics Sonochemistry. 27: 602-608. 
Efecto de la extracción asistida por ultrasonido en la liberación y bioaccesibilidad in vitro de carotenoides, en bebidas elaboradas con mango (Mangifera indica L.) 'Ataulfo'

Taipina, M.S., Lamardo, L. C. A., Camilo-Campos, N., Tavares, M., González, E., Ferrerira-Jorge, L. I. y Aued-Pimentel, S. (2013). Review of literatura: carotenoids, chemical composition and dietary reference intake of buriti fruits. International Journal of Nutrology. 6(3): 102106.

Wassef, L., Wirawan, R., Chikindas, M., Breslin, P, A. S., Hoffman, D. J. y Quadro, L. (2014). $\beta$ carotene-producing bacteria residing in the intestine provide vitamin A to mouse tissues in vivo. Journal of Nutrition. 144(5): 608-613.

Zhang, Y. J., Gan. R. Y., Li, S., Zhou, Y., Li, A. N., Xu, D. P. y Li, H. B. (2015). Antioxidant phytochemicals for the prevention and treatment of chronic diseases. Molecules. 20: 2113821156.

Zou, Y. y Jiang, A. (2016). Effect of ultrasound treatment on quality and microbial load of carrot juice. Food Science and Technology, Campinas. 36(1): 111-115. 